\title{
L'ANALYSE DE COVARIANCE THÉORIE ET APPLICATION DANS L'EXPÉRIMENTATION FORESTIÈRE
}

PAR

\section{R. TOMASSONE}

Ingénieur des Eaux et Forêts

Assistant au Centre National

de Recherches forestières (Station de Biométrie) 



\section{I - INTRODUCTION}

Quand il utilise un plan d'expérience, l'expérimentateur désire contrôler les facteurs qu'il veut étudier de la façon la plus précise: avec les diverses mesures d'un mème caractère il fait une analyse de variance. D'autre part, des renseignements précieux lui sont fournis par les mesures d'autres caractères concomitants. Avec l'ensemble des mesures de ces différents caractères, il peut faire une analyse de variance généralisée (1). Notre objet ici est différent. A partir de ce même ensemble de mesures, nous nous proposons d'étudier un seul caractère, en éliminant l'effet des autres. Prenons le cas simple d'une expérience forestière: on désire comparer la production des peuplements d'une essence particulière soumise à des types d'éclaircie différents. $\mathrm{Si}$ on étudie quatre types d'éclaircie, un bon plan d'expérience consiste à prendre un carré latin $4 \times 4$. L'affectation des modalités d'éclaircie se fait suivant un schéma classique (2). Malgré toutes les précautions, on ne peut empécher une certaine hétérogénéité des arbres soumis aux différentes modalités. Il est donc nécessaire de tenir compte des conditions initiales de l'expérimentation (3).

On peut se demander si dans d'autres cas que celui que nous venons de citer cette analyse ne constitue pas un luxe superflu. En effet, l'objet même de la mise en place d'un plan d'expérience est de contrôler les facteurs qui ne sont pas intéressants à analyser: pour le faire, on distribue les traitements au hasard au sein d'une même unité de comparaison (4). L'analyse de covariance ne devrait done rien apporter de nouveau: ce résultat est toutefois beaucoup plus théorique que réel. Il est bon de tenir compte de ces effets extérieurs et difficilement contrôlables. En utilisant un plan d'expérience et en mesurant les variables concomitantes, le résultat ne pourra être que plus précis.

(1) Cf. Dempstrk (P.A.), op. cit.

(2) Cf. par ex. Vesseresu (A.), op. cit., p. 224-236.

(3) Des expériences de ce type sont en cours d'exécution à la Station Centrale de Sylviculture et de Production, Cf, aussi Ḿackenzie (A.M.), op. cit.

(4) Dans un plan d'expérience en blocs complets ou incomplets, l'unité de comparaison est le bloc. On appelle traitements les facteurs qu'on désire analyser. Dans l'expérience en carré latin citée plus haut les variantes du traitement * intensité d'éclaircie \& sont les différents types d'éclaircie; pour ces définitions $\mathrm{cf}$. Ducué (D.), op. cit., p. 259-260. 


\section{II - THEORIE DE L'ANALYSE DE COVARIANCE}

Nous avons indiqué les points essentiels de l'analyse aussi bien théoriquement que pour les applications: la partie théorique est notée en caractères plus fins; le lecteur qui ne veut voir que l'application peut fort bien sauter les passages correspondants.

\section{1 - Le modèle linéaire utilisé.}

Les modèles mathématiques généralement adoptés dans létude des plans d'expérience sont linéaires. Cela signifie qu'on ne s'intéresse qu'à des variations du premier ordre entre les traitements à étudier et à comparer. Cette hypothèse est logique puisque dans les expériences courantes on cherche à déceler des différences faibles.

De mème lorsqu'on étudie la régression d'une variable dépendante $Y$ en fonction de variables indépendantes $X_{1}$ on adopte un modèle linéaire:

$$
Y=b_{p}+b_{1} X_{1}+b_{2} X_{2}+\ldots b_{p} X_{p}
$$

Il s'agit alors d'une linéarité en fonction des coefficients de régression (1); on peut très bien utiliser comme variable indépendante $X_{1}$ une fonction quelconque de $X_{1}(2)$.

Pour démontrer les résultats généraux que nous appliquerons par la suite à des exemples nous emploierons la notation matricielle maintenant très utilisée même dans le domaine biologique (3).

\section{2 - Notations.}

Supposons qu'on ait effectué n mesures d'un caractère $\mathrm{Y}$, nous pouvons représenter ce résultat par un vecteur a colonne o unique

(1) Cf. Kendal. (M.G.), and Stuakt (A.), op. cit., p. 355 ,

(2) Dans le domaine forestier on utilise couramment des tarifs de cubage dans lesquels le volume est une fonction linéaire du carré de ta circonférence.

(3) Le calcul matriciel par sa grande concision est un outil extrémement efficace; il permet de condenser en une seule formule tout un ensemble d'équations. Pour une étude du calcul matriciel très suffisante pour un biologiste cf. Monjallon (A.), op. cit. ou Vignal. (J.), op. cit. Une matrice sera toujours écrite avec un caractére mral $\mathbf{A}$, il en seta de mème pour un vecteur qui n'est qu'une matrice particulière. Pour plus de détails au sujet de ces notations, cf. Tomassone (R.), op. cit., p. 603-606. 
que nous appellerons vecteur observation et que nous noterons:

$$
\mathbf{Y}=\left\{\begin{array}{c}
y_{1} \\
y_{2} \\
\cdot \\
\cdot \\
\vdots \\
y_{n}
\end{array}\right\}
$$

Sur les n éléments étudiés on a mesuré p autres caractères que nous représenterons par la matrice:

$$
\mathbf{X}=\left\{\begin{array}{cccc}
\mathrm{x}_{11} & \mathrm{x}_{12} & \ldots & \mathrm{x}_{1 \mathrm{p}} \\
\mathrm{x}_{21} & \mathrm{x}_{22} & & \mathrm{x}_{2 \mathrm{p}} \\
\cdot & \cdot & \cdot \\
\cdot & \cdot & \cdot \\
\cdot & \cdot & \cdot \\
\mathrm{x}_{\mathrm{n} 1} & \mathrm{x}_{\mathrm{n} 2} & & \mathrm{x}_{\mathrm{np}}
\end{array}\right\}
$$

cette matrice est évidemment formée par la juxtaposition de p vecteurs "colonne n; chaque vecteur est associé à l'un des p caractères.

Supposons enfin qu'on veuille comparer $t$ traitements, le vecteur traitement est défini par (1):

$$
\Theta=\left\{\begin{array}{c}
\theta_{1} \\
\theta_{2} \\
\cdot \\
\cdot \\
\theta_{t}
\end{array}\right\}
$$

(1) Notons que les composantes de $\Theta$ ne sont pas obligatoirement les paramètres correspondant à un seul facteur contrólé. Pour un plan d'expérience en blocs complets les $\theta$ sont aussi bien les paramètres relatifs aux traitements proprement dits qu'aux blocs. Le test de l'effet des divers facteurs s'effectue après avoir effectué une partition de $\Theta$. 
Le modèle mathématique que nous utiliserons est done:

$$
\mathbf{Y}=\mathbf{A} \Theta+\mathbf{X} \mathbf{B}+\varepsilon
$$

où: A est une matrice $(n \times t)$ donnée dépendant du plan d'expérience (1)

B est un vecteur $(p \times 1)$ dont les $p$ composantes $\beta$ sont les coefficients de régression partielle de $\mathrm{y}$ par rapport aux $\mathrm{x}$

$\varepsilon$ est un vecteur $(n \times 1)$ dont les composantes $\varepsilon_{1}$ sont distribuées normalement avec une moyenne nulle et une variance $\sigma^{2}$.

\section{3 - Calcul des coefficients de régression et des paramètres des traitements.}

On applique le théorème des moindres carrés en cherchant à rendre minimum la quantité :

$$
\mathrm{U}=\sum_{1=1}^{i} \sum^{n} \varepsilon^{2} ;
$$

en langage matriciel on a:

$$
\mathrm{U}=\varepsilon^{\prime} \varepsilon=(\mathbf{Y}-\mathbf{A} \Theta-\mathbf{X} \mathbf{B})^{\prime}(\mathbf{Y}-\mathbf{A} \Theta-\mathbf{X} \mathbf{B})
$$

il faut que la quantité dU soit nulle pour toutes les variations des composantes des vecteurs $\Theta$ et $\mathbf{B}$; on doit donc résoudre le système suivant:

$$
\begin{aligned}
& -\frac{1}{2} \frac{\delta U}{\delta \Theta}=A^{\prime}(\mathbf{Y}-\mathbf{A} \Theta-\mathbf{X} \mathbf{B})=0 \\
& -\frac{1}{2} \frac{\delta U}{\delta \mathbf{B}}=\mathbf{X}^{\prime}(\mathbf{Y}-\mathbf{A} \Theta-\mathbf{X} \mathbf{B})=0
\end{aligned}
$$

soit en développant :

$$
\begin{aligned}
& \mathbf{A}^{\prime} \mathbf{A} \Theta+\mathbf{A}^{\prime} \mathbf{X} \mathbf{B}=\mathbf{A}^{\prime} \mathbf{Y} \\
& \mathbf{X}^{\prime} \mathbf{A} \Theta+\mathbf{X}^{\prime} \mathbf{X} \mathbf{B}=\mathbf{X}^{\prime} \mathbf{Y}
\end{aligned}
$$

Nous notons dès maintenant que la première des équations (II.4) est équivalente à $t$ équations linéaires, la seconde à p. En outre, on peut remarquer que la matrice $\mathbf{X}^{\prime} \mathbf{X}$ est une matrice $(\mathrm{p} \times \mathrm{p})$ dont l'élément $\mathrm{i}, \mathbf{j}$ est:

$$
\sum_{k=1}^{k} x_{k i} x_{k j}
$$

(1) Choisir un plan d'expérience revient done à choisir une matrice $\mathbf{A}$ pour que les paramètres des divers facteurs puissent être déterminés indépendamment. Cf. KeMprhorne (O.), op. cit., p. 59-65. 
Si $\mathrm{i}=j$, cet élément est égal à la somme des carrés des mesures du caractère $\mathrm{i}$; si $\mathrm{i} \neq \mathrm{j}$ il est égal à la somme des produits des mesures du caractère $\mathrm{i}$ par les mesures du caractère $\mathrm{j}$. Il en est de méme pour le vecteur $(\mathrm{p} \times 1)$ $\mathbf{X}^{\prime} \mathbf{Y}$.

Pour résoudre ce système, il est logique d'introduire le vecteur $\mathbf{V}$ qui est la valeur de $\Theta$ lorsque tous les coeíficients de régression sont nuls, soit donc:

$$
\mathbf{A}^{\prime} \mathbf{A} \mathbf{V}=\mathbf{A}^{\prime} \mathbf{Y}
$$

on en déduit immédiatement :

$$
\Theta=\mathbf{v}-\mathbf{W} \mathbf{B}
$$

en posant :

$$
\mathbf{W}=\left(\mathbf{A}^{\prime} \mathbf{A}\right)^{-\mathbf{1}} \mathbf{A}^{\prime} \mathbf{X}
$$

La solution pour $\mathbf{B}$ est alors:

$$
\left(\mathbf{X}^{\prime} \mathbf{X}-\mathbf{X}^{\prime} \mathbf{A W}\right) \mathbf{B}=\mathbf{X}^{\prime} \mathbf{Y}-\mathbf{X}^{\prime} \mathbf{A V} \quad \text { soit : }
$$

$$
\mathbf{E} \mathbf{B}=\mathbf{Q}
$$

Le coefficient de $\mathbf{B}$ au premier membre est la matrice résiduelle

\begin{tabular}{|c|c|c|c|c|c|c|c|c|c|c|c|}
\hline$x^{2}$ & \multicolumn{6}{|c|}{$x_{1} x_{2}$} & \multicolumn{4}{|c|}{$x_{1} x_{0}$} & $x_{1} y$ \\
\hline & $b_{1}$ & + & $\mathrm{PE}$ & $b_{2}$ & & & + & $\mathrm{PE}$ & $b_{D}$ & $=$ & $\begin{array}{c}P E \\
x_{0}, y\end{array}$ \\
\hline $\mathrm{PE}$ & $b_{1}$ & + & $Q E^{2}$ & $b_{2}$ & + & $\ldots$ & + & $\mathrm{PE}^{2}$ & $b_{p}$ & $=$ & $\mathrm{PE}_{\mathrm{E}}$ \\
\hline $\begin{array}{l}\mathrm{x}_{1} \mathrm{x}_{\mathrm{D}} \\
\mathrm{PE}\end{array}$ & $b_{1}$ & + & $\mathrm{PE}^{\mathrm{x}_{2} \mathrm{x}_{\mathrm{r}}}$ & $b_{2}$ & + & * & + & $Q_{\mathrm{E}}^{\mathrm{x}^{2}{ }^{\mathrm{b}}}$ & $b_{0}$ & $=$ & $\mathrm{PE}^{x_{p} y}$ \\
\hline
\end{tabular}
des carrés et des produits. Le vecteur $\mathbf{Q}$ est la somme résiduelle des produits de $\mathrm{Y}$ avec chacune des variables $\mathrm{X}$. On doit résoudre le système d'équations suivant:

Les quantités $Q_{E}^{x_{1}{ }^{2}}$ sont les sommes résiduelles des carrés et les $\mathrm{P}_{\mathrm{E}}^{\mathrm{x}_{1} \mathrm{x}_{j}}$ les sommes résiduelles des produits qui sont calculées à la ligne erreur des tableaux d'analyse de variance (2).

Une fois connus les coefficients de régression, on calcule les valeurs des paramètres $t_{1}$ par (II,6).

(1) On peut voir que $\mathbf{W}$ a une interprétation simple: au caractère $\mathbf{Y}$ correspond un vecteur solution $\mathbf{V}$, aux $p$ caractères représentés par la matrice $\mathbf{X}$ correspond une matrice solution $\mathbf{W}$.

(2) Le système des équations (II,9) n'est que la transcription de l'équation (II, 8 bis), Nous employons ici les notations de Vesseread (A.), op. cit., p. 455. Dès maintenant nous remplaçons les quantités théoriques $\beta$ par les valeurs cstimées b suivant les conventions habituelles. 
On calcule la somme résiduelle ajustée des carrés: c'est la variation totale de $\mathrm{Y}$ à laquelle on soustrait la part de la variation due aux traitements et celle due à la régression:

$$
\begin{aligned}
& \hat{D E}^{2}=\mathbf{Y}^{\prime} \mathbf{Y}-\mathbf{Y}^{\prime} \mathbf{A} \Theta-\mathbf{Y}^{\prime} \mathbf{X} \mathbf{B} \\
& =\mathbf{Y}^{\prime} \mathbf{Y}-\mathbf{Y}^{\prime} \mathbf{A} \mathbf{V}-\left(\mathbf{Y}^{\prime} \mathbf{X}-\mathbf{Y}^{\prime} \mathbf{A} \mathbf{W}\right) \mathbf{B} \\
& =\mathrm{QE}^{\mathrm{y}^{2}}-\mathrm{b}_{1} \mathrm{PE}^{\mathrm{x}_{1} \mathrm{y}} \quad \ldots \quad \mathrm{b}_{2} \mathrm{PE}^{\mathrm{x}_{2} \mathrm{y}}-\ldots \mathrm{b}_{\mathrm{n}} \mathrm{PE}^{\mathrm{PE}_{\mathrm{B}} y}
\end{aligned}
$$

\section{4 -Conditions d'application de l'analyse de covariance.}

Nous avons fait, au moins d'une façon implicite, trois hypothèses dans la démonstration précédente. Pour pouvoir appliquer les résultats de cette démonstration, il faut que ces conditions soient vérifiées; nous avons admis:

a) qu'il existait un vecteur de B commun à tous les traitement. Cette affirmation n'est pas évidente et il peut être nécessaire de vérifier l'égalité des coefficients de régression partielle (1).

b) que les p caractères $\mathrm{X}$ n'étaient pas influencés par les traitements. On ne tient compte de ces caractères dans l'analyse que pour les éliminer; dans la formule (II,2) $\mathbf{X}$ est une valeur fixée qu'on n'ajuste pas à un modèle. On peut toutefois appliquer la méthode dans le cas où les variables à éliminer sont influencées par les traitements; mais il faut être très prudent dans l'interprétation. Cette hypothèse est sûrement vérifiée quand les variables à éliminer sont des variables étudiées avant l'application des traitements.

c) qu'il existait une variance commune autour de la droite ou du plan de régression; cette hypothèse peut ètre vérifiće en utilisant le test de Bartlett (2).

\section{5 - Les hypothèses à tester.}

Elles sont au nombre trois.

IJ.5.1. Intérêt de la régression globale: s'il n'y avait aucune régression tous les coefficients $\beta$ seraient nuls aux erreurs aléatoires près, il n'y aurait donc aucun gain à espérer en employant les p variables. Pour s'en assurer on teste l'hypothèse:

$$
\beta_{1}=\beta_{2} \ldots-\beta_{p}=0
$$

Si elle est exacte la somme résiduelle des carrés $Q_{\mathrm{E}}^{\mathrm{y}^{2}}$ ne diffère $\hat{y}^{2}$

de $\mathrm{D}_{\mathrm{E}}$ que par des variations d'échantillonnage.

(1) Cf, Rno (C.R.), op. cit., p. 112-115.

(2) Cf. Vessereau (A.), p. 157-158. 
II.5.2 Utilisation d'un nombre de variables inférieur à $p$; on peut se demander si après avoir utilisé $\mathrm{p}-1$ variables, il est utile de prendre encore la dernière. Si on veut voir l'utilité de la variable $\mathrm{X}_{\mathrm{p}}$ on teste l'hypothèse

$$
\beta_{p}=0
$$

Si cette hypothèse est exacte, on a pour calculer les autres $\beta$ un système de rang $\mathrm{p}-1$ dont les solutions sont $\beta_{1}^{\prime}, \beta_{2}^{\prime} \ldots \beta_{p-1}^{\prime}$. La nouvelle somme résiduelle ajustée des carrés est de:

$$
D_{E}^{\hat{y}^{2}}=Q E^{y^{2}}-b_{1}^{\prime} Q_{E}^{x_{1} y}-\ldots b_{D-1}^{\prime} Q E^{x_{p-1} y}
$$

La comparaison entre:

$$
D^{\prime} \hat{E}^{2} \text { et } \hat{D}^{\hat{y}^{2}}
$$

\begin{tabular}{|c|c|c|c|c|}
\hline Origine de la variation & Somme des carrés & d.1. & Carré moyen & F \\
\hline Régression en $x_{1}, x_{y}, \ldots, x_{n}$ & $b_{1}{\stackrel{x}{x_{1} y}}^{P_{n}}+\ldots b_{n} P_{n}^{x_{n} y}$ & $\mathrm{p}$ & & \\
\hline $\begin{array}{l}\text { Régression en } x_{1}, x_{2} \ldots x_{n-1} \\
\text { Apport de } x_{n} \text { après utilisation } \\
\text { des } p-1 \text { premières }\end{array}$ & 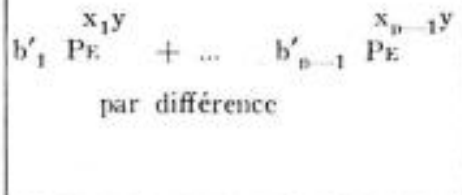 & $\begin{array}{c}p-1 \\
1\end{array}$ & $\mathrm{~S}_{\mathrm{p}}^{2}$ & $\frac{\mathrm{S}^{2} \mathrm{~g}}{\mathrm{~S}_{\mathrm{y} 2}}$ \\
\hline : & : & : & ' & \\
\hline 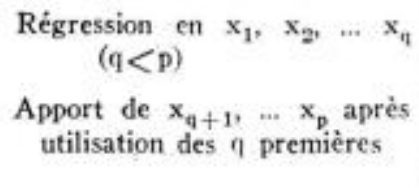 & 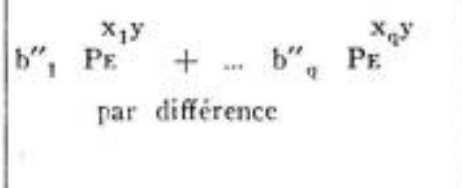 & $\stackrel{\mathrm{q}}{\mathrm{p}-\mathrm{q}}$ & $S_{p D-1}^{2} \cdots p-q$ & $\frac{\mathrm{S}_{\mathrm{p1}}^{2} \cdots \mathrm{pq}}{\mathrm{S}^{\mathrm{y}^{2}}}$ \\
\hline : & : & : & & \\
\hline Somme résiduelle ajustée & $\frac{y^{2}}{Q E}-b_{1}{ }^{x_{1} y}-\ldots b_{b} P_{E}^{x_{b} y}$ & $\mathrm{nE}$ & $\mathrm{S}_{y 2}$ & \\
\hline
\end{tabular}

permet de vérifier l'hypothèse ci-dessus.

On peut résumer ces résultats dans le tableau II.5.1 suivant :

Tableau II.5.1. Test de l'effet des caractères concomitants. 
11.5.3 - Test de leffet des traitements: Supposons qu'on désire tester l'hypothèse tous les $\theta_{1}$ sont égaux. Dans ce cas, la somme des carrés vaut:

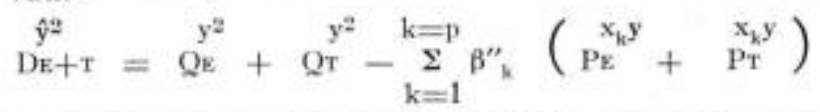

Dans cette égalité, les $\beta^{\prime \prime}$ sont les solutions du système noté sym4. $\hat{y}^{2} \quad \hat{y}^{2}$ boliquement $\mathrm{E}+\mathrm{T}$; la différence $\mathrm{DE}_{+} \mathrm{T}-\mathrm{DE}$ permet de calculer la somme des carrés traitement ajustée, et de tester l'hypothése d'égalité des $\theta_{\text {; }}$.

\section{I. 6 - Classement des traitements. Comparaison des moyennes ajustées.}

On appelle moyenne ajustée du traitement $\mathrm{i}$ et on note $\hat{y}_{1}$ la valeur quaurait eu $\bar{y} i$ si les valeurs $\mathrm{x}$ avaient été les mèmes pour tous les traitements. On rapporte habituellement les valeurs y aux valeurs moyennes des $\mathrm{x}(1)$, on a alors

$$
\hat{y}_{1}=\tilde{y}_{1}-\underset{k=1}{\mathrm{i}=\mathrm{p} b_{\mathrm{k}}}\left(\overline{\mathrm{x}_{\mathrm{k} 1}}-\overline{\mathrm{x}_{\mathrm{k}}}\right)
$$

où les quantités $\overline{\mathrm{x}}_{\mathrm{ko}}$ sont les moyennes des caractères $\mathrm{x}$.

La différence entre deux moyennes ajustées est donc:

$$
d_{i j}=\left|\hat{y}_{i}-\hat{y}_{j}\right|=\left|\bar{y}_{1}-\bar{y}_{j}-\Sigma_{k} b_{k}\left(\overline{x_{k i}}-\overline{x_{k j}}\right)\right|
$$

La variance de cette différence fait intervenir les valeurs des caractères concomitants, il est en général préférable d'avoir une valeur moyenne de la variance d'une moyenne ajustée; cette variance est calculée par la formule:

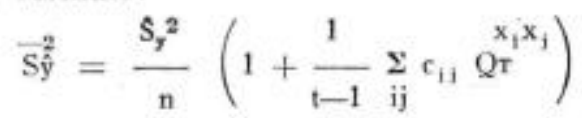

où

$\widehat{S}_{y}{ }^{2}$ est la variante résiduelle moyenne

$\mathrm{x}_{1} \mathrm{x}_{\mathrm{j}}$ : les éléments de la matrice de la somme des carrés et des produits relative au facteur traitement

$c_{11}$ : les éléments de la matrice $\mathbf{E}^{-\mathbf{1}}$

$\mathrm{t}$ : le nombre de variantes du traitement à étudier

$\mathrm{n}$ : le nombre de mesures d'une variante.

(1) L'interprétation géométrique est simple: s'il existe une ligne moyenne de régression commune (cette ligne peut étre une droite, un plan ou un hyperplan selon le nombre de variables concomitantes). On trace des parallèles à cette ligne par les points moyens de chaque variante des traitements: l'intersection de cette ligne avec la parallèle à Oy menée par le point moyen commun donne la valeur de la moyenne ajustée. 
Dans le cas où on a une seule variable à éliminer la formule se simplifie et devient (1):

$$
\overline{\mathrm{S}_{y}^{2}}=\frac{\mathrm{S}_{y}{ }^{2}}{\mathrm{n}}\left(1+\frac{\mathrm{QT}^{* 2}}{(\mathrm{t}-1) Q_{\mathrm{E}}^{\mathrm{x}^{2}}}\right)
$$

C'est l'écart type correspondant qu'on doit utiliser pour comparer les moyennes par le test de Duncan (2).

\section{7 - Gain apporté par l'analyse de covariance.}

On chiffre ce gain en faisant le rapport de la variance avant ajustement à la variance après ajustement (3). C'est donc une quantité liée at coefficient de corrélation multiple qui mesure la fraction de la variance totale de $\mathrm{Y}$ qui peut être imputée à la liaison entre $\mathrm{Y}$ et les variables $\mathrm{X}_{1}, \mathrm{X}_{2} \ldots \mathrm{X}_{\mathrm{p}}$.

\section{III - EXEMPLES D'ANALYSES DE PLANS ORTHOGONAUX}

\section{1 - Analyse à un seul facteur contrôlé.}

(Bouvarel. (P.) et Lemoine. Provenances d'épicéas T 357. Dossier 63-54)

Pour comparer quatre provenances d'épicéas on voulait comparer le caractère: poids de cent graines. Ce poids n'est sûrement pas le mème si les graines sont pleines ou vides: il est done normal de tenir compte de ce facteur et de comparer les poids moyens de cent graines des quatre provenances en supposant qu'on les a toutes ramenées au même pourcentage de graines pleines. C'est ce dernier caractère qu'on désire donc éliminer (4).

Nous appelons:

X: la transformée du pourcentage par la transformation $\operatorname{Arcsin} \sqrt{\%}(5)$

$\mathrm{Y}:$ le poids de cent graines en mg.

L'analyse de covariance se fait alors en trois étapes.

III.1.1. Calculs préliminaires: ils sont indiqués au tableau III.1.1; on calcule les sommes des mesures pour $\mathrm{X}$ et pour $\mathrm{Y}$, ainsi

(1) Cf. Snepecor (G.W.), op. cit., p. 444 et Cochran (W.T.) et Cox (G.T.), op. cit., D. 79.

(2) Cf. Rrves (M.), op. cit., pour lapplication de ce test plus surr que le test de Student.

(3) Il ne faut pas oublier que le nombre de degré de liberté de la variance après ajustement est diminué de p. unités.

(4) Les quatre provenances appartiennent à deux régions différentes: la Haute-Savoie pour Morzine (MORZ) et Monriond (MONR); le Jura pour Molltunes (MOLU) et Premanon (PREM). Sur chaque provenance on a prélevé dix échantillons.

(5) Cette transformation est nécessaire pour stabiliser la variance des données exprimées en pourcentage. 
que les sommes des carrés $\mathrm{X}^{2}$ et $\mathrm{Y}^{2}$; enfin les sommes des produits XY. On remplit alors sans aucune difficulté les colonnes (1) à (5), les lignes MORZ, MONR, MOLU, PREM et « totale $n$ du tableau III.1.2. Par exemple pour

$\begin{array}{ccc}\text { MORZ: } & 39483,585-38850,289= & 633,296 \\ & 369630,67-364194,19=5436,48 \\ \text { totale : } & 3499235,0-3414064,9=85170,1 \\ & 156870,652-152815,335=4055,317 \\ & \text { etc... }\end{array}$

III.1.2 Hypothèses à vírifier: Les conditions nécessaires imposées at paragraphe 11.4 sont:

a) Existence d'un coefficient de régression unique: puisqu'il n'y a qu'une variable à éliminer le vecteur $\mathbf{B}$ est un vecteur $(1 \times 1)$ don: un nombre. L'équation (II.8 bis) est donc très simple puisque:

$$
3458,874 b_{1}=28739,34
$$

le résultat est donné en colonne (6). Nous avons aussi calculé les valeurs des coefficients de régression interne pour chaque provenance. L'hypothèse de l'égalité est assez vraisemblable, d'après la figure III.1.1., on peut le vérifier rigoureusement en calculant les variations résiduelles ajustées pour chaque provenance. Ia somme de ces quantités est inférieure à la variation résiduelle; la différence est une somme de carrés à trois degrés de liberté qui permet de tester l'hypothèse d'égalité. Ici, comme on potvait le supposer. rien ne permet de contredire cette hypothèse.

b) Le caractère concomitant n'est pas influencé par les traitements: une analyse de variance sur le caractère $\mathrm{X}$ permet de l'affirmer.

c) Les variances résiduclles internes sont égales: un test de Bartlett sur les quatre carrés moyens de la colonne (10) permet de l'affirmer.

III.1.3 Analyse de covariance proprement dite: il suffit d'utiliser le tableau III.1.2 où sont déjà remplies les lignes et les colonnes utilisées au paragraphe précédent. La part de la variation résiduelle qui n'est due qu'à la régression se calcule à la ligne erreur par

$$
\text { b. }_{1} \mathrm{P}_{\mathrm{E}}^{\mathrm{xy}}-8,3089 \times 28739,34-238792,3
$$

Cette quantité soustraite à la variation résiduelle de $\mathrm{Y}$ avant ajustement permet de calculer la somme résiduelle ajustée des carrés $\hat{\mathrm{y}}^{2}$

$D_{\mathrm{E}}=197501,9$ et le carré moyen résiduel ajusté à 35 degrés de liherté. Pour tester l'hypothèse de l'égalité des moyennes du poids de 100 graines pour les quatre provenances, on effectue exactement les mếmes opérations que ci-dessus pour la quantité " Er. 


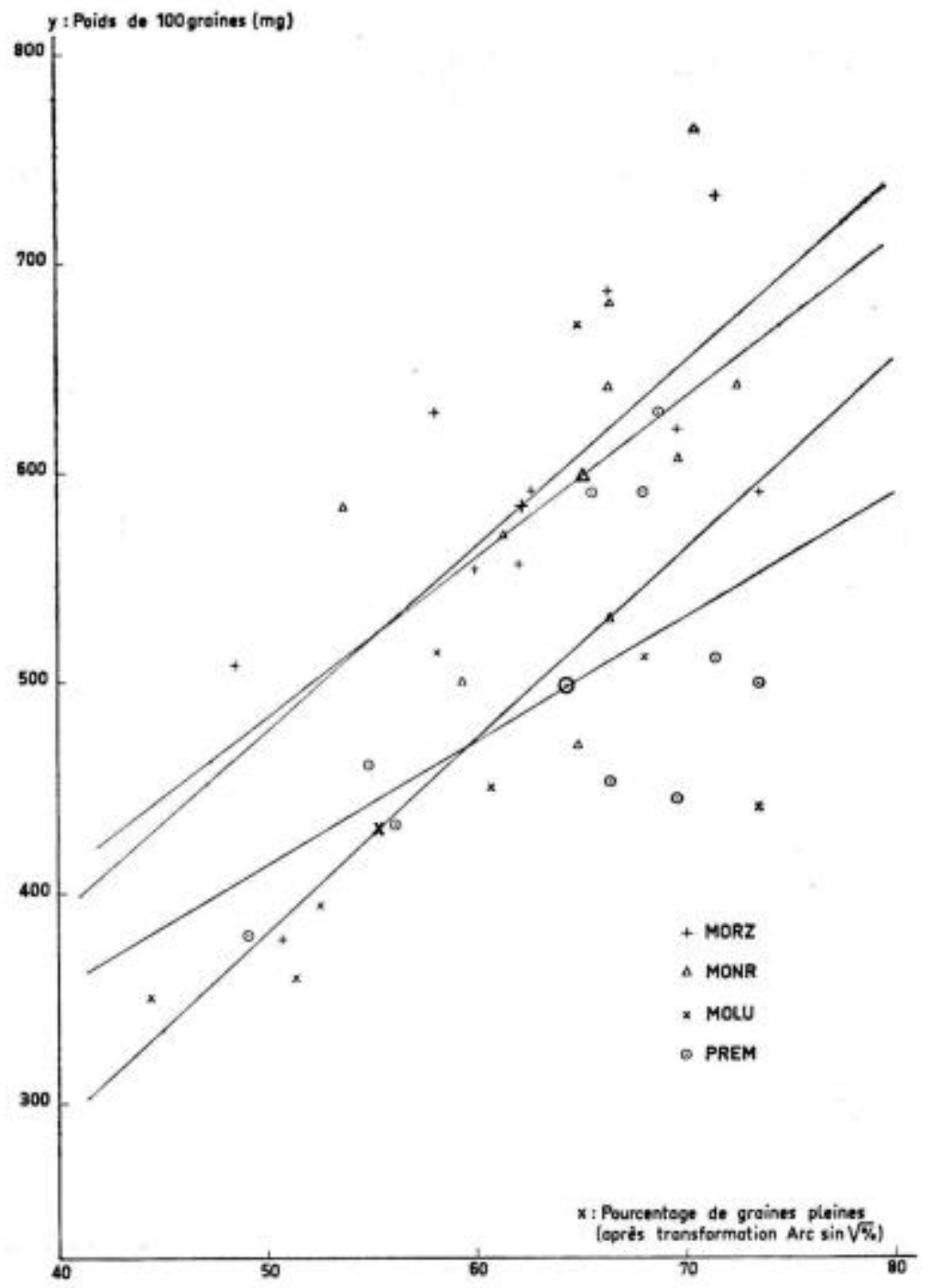

Figure III.1.1: Relation entre le poids de 100 graines et le pourcentage de graines pleines (les quatre points moyens sont indiqués par des traits plus forts). 


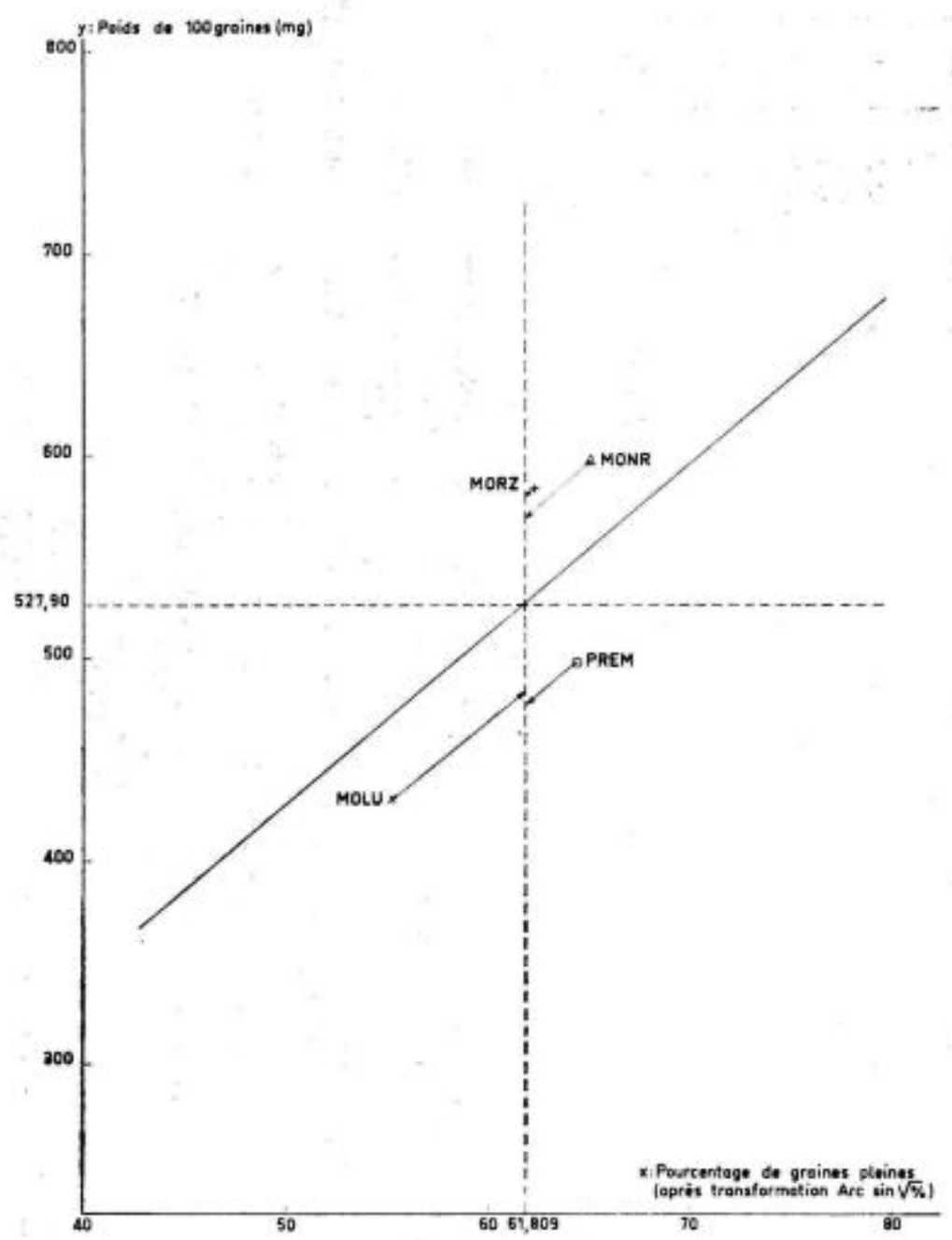

Figure III.1.2: Ajustement graphique du poids de 100 graines. 


\begin{tabular}{|c|c|c|c|c|c|c|c|c|c|}
\hline $\begin{array}{c}\text { (1) } \\
\text { Origine } \\
\text { de la variation }\end{array}$ & (2) & $\begin{array}{l}\text { (3) } \\
x^{2}\end{array}$ & (4) & $\begin{array}{l}\text { (5) } \\
y^{\prime \prime}\end{array}$ & $(6)=\frac{(4)}{b_{5} \times}$ & $\begin{array}{l}\quad(7) \\
=(6) \times(4) \\
\text { Part de la } \\
\text { régression }\end{array}$ & $\begin{aligned} & \text { (8) } \\
&=(5)-(7) \\
& \text { Eeart par } \\
& \text { rapport à la } \\
& \text { régression }\end{aligned}$ & $\begin{array}{l}\text { (9) } \\
\text { d.1. }\end{array}$ & $10=\frac{(8)}{(9)}$ \\
\hline $\begin{array}{l}\text { Morzine } \ldots . . . \\
\text { Monriond } \ldots . . . \\
\text { Mollunes } \ldots . . . \\
\text { Premanon } \ldots . .\end{array}$ & $\begin{array}{l}9 \\
9 \\
9 \\
9\end{array}$ & $\begin{array}{r}633,296 \\
289,208 \\
1940,037 \\
596,333\end{array}$ & $\begin{array}{r}5436,48 \\
2230,13 \\
17616,13 \\
3456,60\end{array}$ & $\begin{array}{r}85170,1 \\
70258,1 \\
222029,6 \\
58836,4\end{array}$ & $\begin{array}{l}8,5844 \\
7,7112 \\
9,0803 \\
5,7964\end{array}$ & $\begin{array}{r}46668,9 \\
17197,0 \\
159959,7 \\
20035,0\end{array}$ & $\begin{array}{l}38501,2 \\
53061.1 \\
62069,9 \\
38800,6\end{array}$ & $\begin{array}{l}8 \\
8 \\
8 \\
8\end{array}$ & $\begin{array}{l}4812,65 \\
6632,64 \\
7758,74 \\
4850,08\end{array}$ \\
\hline & & & & & & & $\begin{array}{r}192432,8 \\
(5069,1)\end{array}$ & $\begin{array}{r}32 \\
3\end{array}$ & $\begin{array}{l}6013,53 \\
1689,70\end{array}$ \\
\hline $\begin{array}{l}\text { Erreur } . . . . . . . \\
\text { Entre traitements }\end{array}$ & $\begin{array}{l}36 \\
3\end{array}$ & $\begin{array}{l}3458.874 \\
(597.443)\end{array}$ & $\begin{array}{l}28739,34 \\
(8190,75)\end{array}$ & $\begin{array}{c}436294,2 \\
(186091,4)\end{array}$ & 8.3089 & 238792,3 & $\begin{array}{l}197501,9 \\
(88579,8)\end{array}$ & $\begin{array}{r}35 \\
3\end{array}$ & $\begin{array}{r}5642,91 \\
29526,60\end{array}$ \\
\hline Totale ........ & 39 & $405,5,317$ & 36930,09 & 622385,6 & 9,1065 & 336303,9 & 286081,7 & 38 & \\
\hline
\end{tabular}

Hypothèses qu'il est possible de tester d'après le tableau ci-dessus avant analyse de covariance:

- Egalité des coefficients de régression: exacte car la valeur ${ }_{\text {de }}^{3} \mathrm{~F}=\frac{1689,70}{6013,53}$ n'est pas significative.

- Egalité des variances résiduelles de chaque provenance: par test de Bartlett sur les 4 variances (col. (10)) $\quad \chi^{2}=1,07$ à 3 d.1. a une probabilité 0,80 d'être dépassée.

- Le caractìre $X$ n'est pas influencé par les traitements: ${ }_{36}^{3} \mathrm{~F}=\frac{(597,443): 3}{(3458,874): 36}=\frac{199,15}{96,08}=2,07 \mathrm{NS}$

L'analyse de covariance proprement dite permet de tester l'égalité des moyennes ajustées $\mathrm{Y}$

$$
\begin{gathered}
{ }_{35}^{3} \mathrm{~F}=\frac{29526,60}{5642,91}=5,23^{* *} \\
\text { En outre, on peut faire le test global de la régression }{ }_{35}^{1} \mathrm{~F}=\frac{238792,3}{5642,91}=42,32^{* *}
\end{gathered}
$$

Tableau III.1.2. Analyse de covariance : Poids de $100 \mathrm{graines}(\mathrm{Y})$ ajustés (les résultats entre parenthèses sont calculés par différence). 
III.1.4 Comparaison des moyennes: on présente les calculs de la façon suivante:

\begin{tabular}{|c|c|c|c|c|c|}
\hline $\begin{array}{c}\text { (1) } \\
\text { Provenance }\end{array}$ & $\begin{array}{l}(2) \\
\vec{x}_{i o}\end{array}$ & $\begin{array}{c}(3) \\
\bar{x}_{10}-x_{1}= \\
\bar{x}_{\infty 0}\end{array}$ & $\begin{array}{c}\text { (4) } \\
b_{1} \times \delta x_{1}\end{array}$ & $\begin{array}{l}(5) \\
\bar{y}_{10}\end{array}$ & $\begin{array}{c}(6)=(5)-(4) \\
\hat{y}_{i 0}=\bar{y}_{t 0}-b_{1} \delta x_{i}\end{array}$ \\
\hline MORZ & 62,330 & 0,521 & 3,329 & 584,3 & 580,0 \\
\hline MONR & 65,147 & 3,338 & 27,735 & 598,7 & 571,0 \\
\hline MOLU & 55,364 & $-6,445$ & $-53,551$ & 430,2 & 483,8 \\
\hline PREM & 64,396 & 2,587 & 21,495 & 498,4 & 476,9 \\
\hline Total & & 0,001 & 0,008 & & \\
\hline Moyenne & $=61,8$ & \multicolumn{4}{|c|}{$\bar{y}_{00}=527,9$} \\
\hline
\end{tabular}

Tableau III.1.3. Calcul des moyennes $y$ ajustées $\left(b_{1}=8,3089\right)$

La représentation graphique de ce calcul est très simple sur les figures III.1.1 et III.1.2. Sur la première l'ensemble des trentehuit points a été transcrit (1). Sur la seconde seuls les points représentatifs des moyennes ont été indiqués: lajustement revient simplement à tracer des parallèles à la droite de régression résiduelle à partir des points moyens: la valeur de la moyenne ajustée se lit donc à l'intersection de ces droites avec la parallèle à laxe $\mathrm{Y}$ menée par le point $\mathrm{X}=61,809$ (2). La comparaison des moyennes deux à deux s'effectue comme dans une analyse de variance classique. Pour pouvoir appliquer les résultats du test de Duncan, on calcule d'abord la variance moyenne ajustée de la moyenne d'un traitement

$$
\mathrm{S}_{\hat{y}_{x}}{ }_{x}-\frac{5642,91}{10}\left(1+\frac{597,44,3}{3 \times 3458,874}\right)-579,865
$$

soit un écart type $\overline{\mathrm{S} \hat{y}_{\mathrm{x}}}-24,080$

(1) Deux des points représentatifs de la provenance MOLU n'ont pas été indiqués: le premier et le quatrième.

(2) L'analyse de la covariance revient donc à étudier les dispersions des points moyens par rapport à une droite résiduelle commune. 
- On en déduit les plus petites différences significatives (1) entre deux, trois ou quatre moyennes ajustées aux seuils $5 \%$ et $1 \%$.

$\begin{array}{lll}\mathrm{p} & \mathrm{R}(5 \%) & \mathrm{R}(1 \%) \\ 2 & 69,1 & 92,7 \\ 3 & 72,7 & 96,8 \\ 4 & 74,8 & 99,5\end{array}$

et enfin la comparaison des moyennes proprement dite:

\begin{tabular}{|c|l|c|c|c|c|c|}
\hline $\mathrm{i}$ & Provenance & $\hat{y}_{10}$ & $\hat{y}_{10}-\hat{y}_{40}$ & $\hat{y}_{10}-\hat{y}_{30}$ & $\hat{y}_{i 0}-\hat{y}_{20}$ & $\hat{y}_{10}-\dot{y}_{10}$ \\
\hline 1 & MORZ & 580,0 & $103,1^{* *}$ & $96,2^{*}$ & 9,0 & 0 \\
2 & MONR & 571,0 & $94,1^{*}$ & $87,2^{*}$ & 0 & \\
3 & MOLU & 483,8 & 6,9 & 0 & & \\
4 & PREM & 476,9 & 0 & & & \\
\hline
\end{tabular}

Tableau III.1.4. Comparaison des moyennes ajustées.

On voit donc qu'on peut établir les groupements suivants aux deux seuils choisis:

$5 \%$
$1 \%$

MORZ MONR MOLU PREM

III.1.5 Gain apporté par lanalyse de covariance: dans ce cas particulier la liaison étant forte entre les deux caractères, le gain est important en effet:

sans utiliser la régression, le carré résiduel vatut

$$
\mathrm{S}_{y}{ }^{2}=\frac{436294,2}{36}=12119,28 \text { à } 36 \text { d.l. }
$$

Après ajustement il vaut 5642,91 , soit un rapport 2,15 ou $215 \%$. Le gain apporté par cette analyse est sensiblement égal à celui qu'on aurait obtenu en doublant le nombre de mesures.

\section{2 - Analyse d'une expérience factorielle.}

(ARBonnier (P.): étude de destruction de fougère par divers produits chimiques: forêt de Granvillers. Dossier 63.22)

Il s'agissait de comparer l'action de quatre produits chimiques sur la croissance de la fougère aigle (Pteridium aquilimum). Ces produits ont été utilisés à deux reprises pour qu'on puisse déceler une influence possible de la date d'application. En plus, un témoin était introduit dans l'expérience pour pouvoir comparer l'ensemble des

(1) Dans la littérature anglaise ces quantités sont appelées les ranges: : 


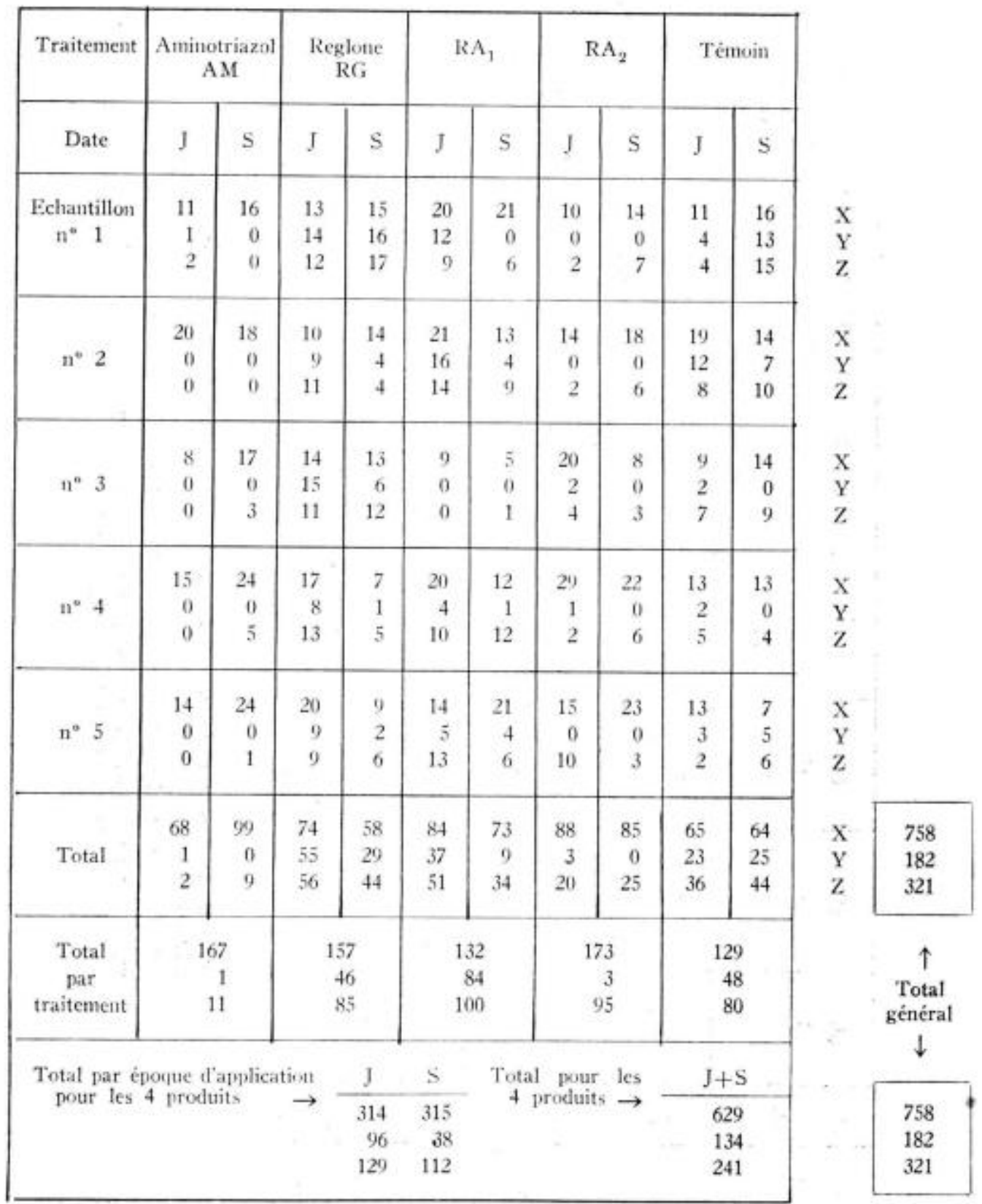

Tableau III.2.1. Résultats de l'expérience de destruction de fougères en forêt de Granvilliers. 


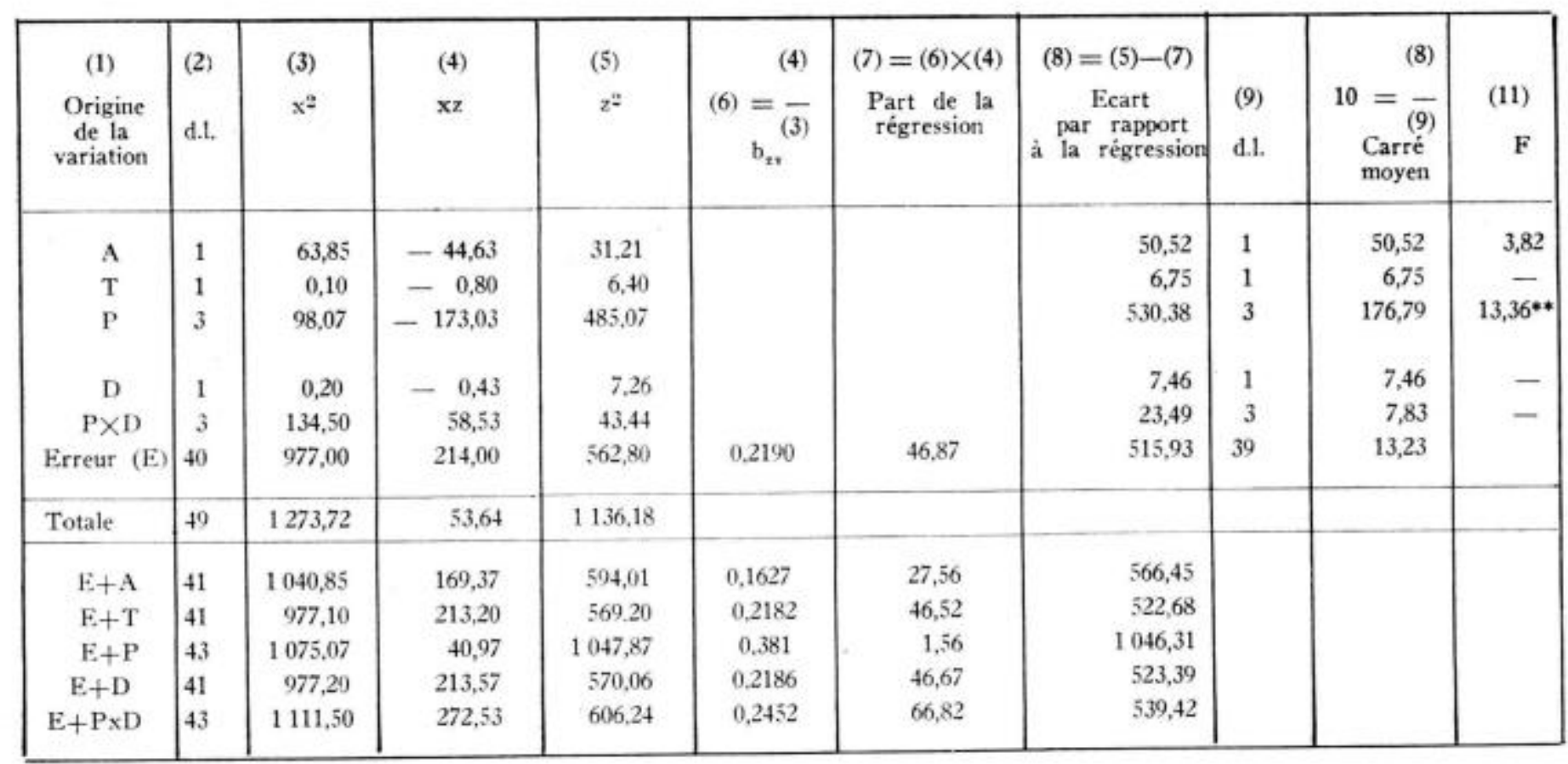

A : entre témoins et traitements; $\mathrm{T}$ : entre témoins; $\mathrm{P}$ : entre produits chimiques;

D: entre époques d'application; PxD: interaction produit $x$ époques.

Tableau III.2.2. Analyse de covariance dans une expérience factorielle (destruction de fougère en forêt de Granvilliers) 
produits chimiques. Sur chaque surface attribuée à un traitement on a pris au hasard cinq surfaces unitaires et on a compté le nombre de crosses de fougères (1):

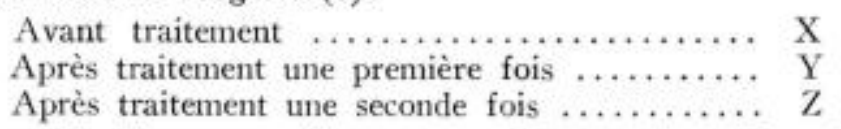

Nous n'étudions pour l'instant que les caractères $\mathrm{X}$ et $\mathrm{Z}$ qui correspondent aux lignes 1 et 3 du tableau III.2.1 (2). A l'aide de ce tableau, on calcule sans difficulté les parts relatives à chaque variation dans l'analyse de covariance du tableau III.2.2: pour tous les calculs de l'analyse de covariance, il faut opérer comme dans une analyse de variance classique: il suffit de se souvenir que toutes les opérations sur les carrés d'une variable $x_{1}$ sont remplacées par des opérations sur les produits des variables $x_{1} x_{1}$.

Comme au paragraphe précédent, on calcule le coefficient de régression résiduelle à la ligne erreur du tableau III.2.2. Pour testé laction des divers traitements et l'interaction entre les produits chimiques et la date d'application, on fait les mèmes opérations que celles du paragraphe précédent sur l'unique test possible de l'expérience " traitement + erreur " (3). Le test des différents facteurs se trouve en colonne 11 . On voit que seuls les produits chimiques ont des effets significativement différents (4).

Pour le calcul des moyennes ajustées, on présente les calculs comme au paragraphe III.1.4:

\begin{tabular}{|c|c|c|c|c|c|}
\hline $\begin{array}{l}\text { (1) } \\
\text { Traitement }\end{array}$ & $\begin{array}{l}(2) \\
\bar{x}_{f 0}\end{array}$ & $\delta \mathrm{x}_{1}=\overline{\mathrm{x}}_{10}-\mathrm{x}_{\mathrm{oa}}$ & $\begin{array}{l}(4) \\
b+8 x_{1}\end{array}$ & $\frac{(5)}{\bar{Z}_{10}}$ & $\begin{array}{l}(6)=(5)-(4) \\
\hat{Z}_{10}=\bar{Z}_{10}-b_{1} 8 x_{1}\end{array}$ \\
\hline $\begin{array}{l}\mathrm{AM} \\
\mathrm{RG} \\
\mathrm{RA} \\
\mathrm{RA}_{2}\end{array}$ & $\begin{array}{l}16,700 \\
13,200 \\
15,700 \\
17,300\end{array}$ & $\begin{array}{r}0,975 \\
-2,525 \\
-0,025 \\
1,575\end{array}$ & $\begin{array}{r}0,214 \\
-0,553 \\
-0,005 \\
0,345\end{array}$ & $\begin{array}{r}1,100 \\
10,000 \\
8,500 \\
4,500\end{array}$ & $\begin{array}{r}9,886 \\
10,553 \\
8,505 \\
4,155\end{array}$ \\
\hline Total & & 0,000 & 0.001 & 24,100 & 24,099 \\
\hline Moyenne & 15,725 & & & 6,025 & \\
\hline
\end{tabular}

Tableau III.2.2, Calcul des moyennes $z$ ajustées (destruction de fougère en forèt de Granvilliers)

(1) Non comprises les crosses sèches.

(2) On peut s'assurer de la normalité de la distribution du nombre de crosses $\mathrm{X}$ par un test graphique: Cf. Tomassone (R.), op. cit. On trouve que rien ne s'oppose à admettre que le caractère $\mathrm{X}$ est distribué normalement avec une moyenne $\mathrm{m}=15,2$ et un écart type $\mathrm{s}=5,1$.

(3) Dans ce cas particulier on avait: \& traitement + erreur $>=$ variation totale, Ici la variation totale n'intervient plus directement.

(4) II n'y a pas de différence entre le témoin et l'ensemble des traitements car la moyenne de ces derniers est sensiblemnt égale à celle du témoin: le réglone et $\mathrm{RA}_{1}$ unt moins d'effet que le témoin. 


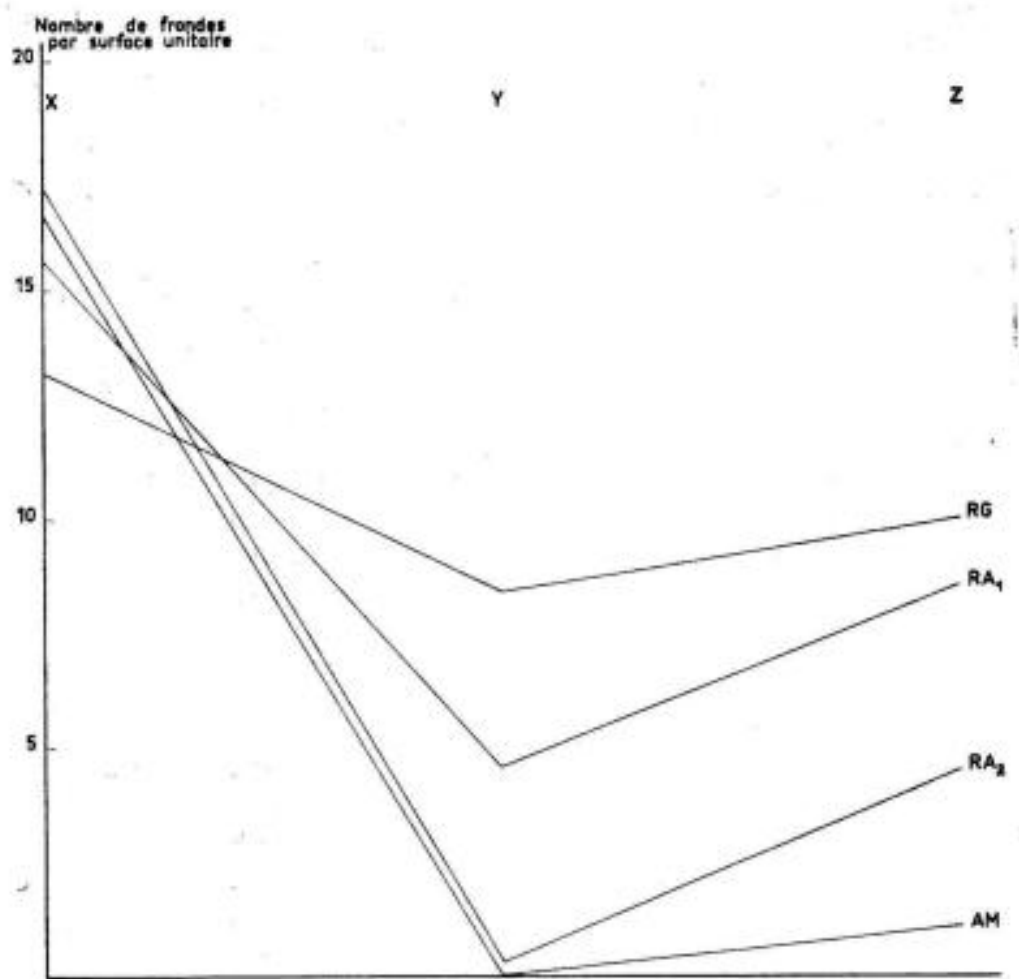

Figure III.4.1: Evolution dans le temps du nombre de frondes de fougères sur des surfaces traitées par quatre produits chimiques (Des. truction de fougère en forèt de Granvillers). 
La variance d'une moyenne ajustée:

$$
\begin{gathered}
\overline{\mathrm{S}}_{\hat{\mathbf{z}}, \mathrm{x}}^{2}=\frac{13,23}{10} \\
\overline{\mathrm{S}_{\hat{\mathrm{z}}, \mathrm{x}}}=1,282
\end{gathered}
$$

La comparaison de ces moyennes par le test de Duncan permet de faire les groupements suivants au seuil de probabilité $5 \%$ :

\begin{tabular}{cccc}
$\mathrm{AM}$ & $\mathrm{RA}_{2}$ & $\mathrm{RA}_{1}$ & $\mathrm{RG}$ \\
$(0,886)$ & $(4,155)$ & $(8,505)$ & $(10,553)$ \\
\hline
\end{tabular}

$$
\begin{aligned}
& \text { Le gain apporté par l'analyse de covariance est ici } \frac{14,07}{13,23}-1,06 \\
& \text { soit } 106 \% \text {. }
\end{aligned}
$$

\section{3 - Analyse d'une expérience en blocs complets avec deux variables concomitantes.}

(LANier (L.): Etude de la réaction de Pinus sylvestris L. à Lophodermium pinastri (Schrad) Chev. après application de différents produits chimiques et anticrytogamiques. Dossier $63.20)$.

Cette expérience, implantée en forêt de Haguenau avec un dispositif en blocs complets comportant quatre répétitions, avait pour but de comparer l'effet de différents produits chimiques et anticryptogamiques (1). Dans chaque parcelle unitaire, cinquante plants ont été échantillonnés. On a mesuré sur chacun:

- la hauteur atteinte avant traitement en $\mathrm{cm}$............ $\mathrm{X}_{1}$

- l'intensité d'attaque avant traitement avec une note pouvant être 1,2 ou 3 , fonction de l'état sanitaire du plant (1 - sain, 2 - légèrement touché, 3 - très touché).

- l'intensité d'attaque après traitement avec la mème nota-

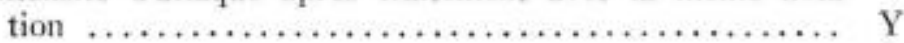

(1) Cette étude faite par M. LAxirR en collaboration avec M. Leroy, ingénieur principal à Haguenau, r'est pas encore terminée. Les premiers résultats ne sont utilisés ici que pour illustrer une méthode et non pour tirer des conclusions d'ordre biologique. 


\begin{tabular}{|c|c|c|c|c|c|c|}
\hline $\begin{array}{l}\text { RLES'S } \\
\text { FRAITEMENTS }\end{array}$ & 1 & 2 & 3 & 4 & Total & Moyenne \\
\hline 1 & $\begin{array}{r}73,08 \\
2,72\end{array}$ & $\begin{array}{r}72,76 \\
2,10\end{array}$ & $\begin{array}{r}66,78 \\
2,28\end{array}$ & $\begin{array}{r}59,80 \\
2,24\end{array}$ & $\begin{array}{r}272,42 \\
9,34\end{array}$ & $\begin{array}{r}68,105 \\
2,335\end{array}$ \\
\hline 2 & $\begin{array}{r}78,34 \\
2,82\end{array}$ & $\begin{array}{r}72,84 \\
2,46\end{array}$ & $\begin{array}{r}69,24 \\
2,34\end{array}$ & $\begin{array}{r}67,28 \\
2,06\end{array}$ & $\begin{array}{r}287,70 \\
9,68\end{array}$ & $\begin{array}{r}71,925 \\
2,420\end{array}$ \\
\hline 3 & $\begin{array}{r}63,16 \\
2,44\end{array}$ & $\begin{array}{r}70,36 \\
2,08\end{array}$ & $\begin{array}{r}49,90 \\
2,60\end{array}$ & $\begin{array}{r}74,40 \\
2,24\end{array}$ & $\begin{array}{r}257,82 \\
9,36\end{array}$ & 1,955 \\
\hline 4 & $\begin{array}{r}81,12 \\
2,16\end{array}$ & $\begin{array}{r}59,26 \\
2,36\end{array}$ & $\begin{array}{r}63,82 \\
2,46\end{array}$ & $\begin{array}{r}64,24 \\
9,84\end{array}$ & $\begin{array}{r}268,44 \\
9,84\end{array}$ & $\begin{array}{r}67,110 \\
2,460\end{array}$ \\
\hline 5 & $\begin{array}{r}74,58 \\
2,54\end{array}$ & $\begin{array}{r}68,44 \\
1,94\end{array}$ & $\begin{array}{r}\$ 8,50 \\
2,56\end{array}$ & $\begin{array}{r}76,04 \\
2,56\end{array}$ & $\begin{array}{r}277,56 \\
9,60\end{array}$ & $\begin{array}{r}69,390 \\
2,400\end{array}$ \\
\hline 6 & $\begin{array}{r}65,04 \\
2,30\end{array}$ & $\begin{array}{r}62,06 \\
1,86\end{array}$ & $\begin{array}{r}61,94 \\
2,04\end{array}$ & $\begin{array}{r}62,06 \\
2,08\end{array}$ & $\begin{array}{r}251,10 \\
8,28\end{array}$ & $\begin{array}{r}62,775 \\
2,070\end{array}$ \\
\hline Total & $\begin{array}{r}435,32 \\
15,58\end{array}$ & $\begin{array}{r}405,72 \\
12,80\end{array}$ & $\begin{array}{r}370,18 \\
14,28\end{array}$ & $\begin{array}{r}403,82 \\
13,44\end{array}$ & $\begin{array}{r}1615,04 \\
56,10\end{array}$ & \\
\hline Moyenne & $\begin{array}{r}72,55 \\
2,59\end{array}$ & $\begin{array}{r}67,62 \\
2,13\end{array}$ & $\begin{array}{r}61,69 \\
2,38\end{array}$ & $\begin{array}{r}67,30 \\
2,24\end{array}$ & & $\begin{array}{r}67,293 \\
2,338\end{array}$ \\
\hline
\end{tabular}

Tableau III.3.1. Résultats de l'expérience sur Lophodermium pinastri (Schard) Chev. 


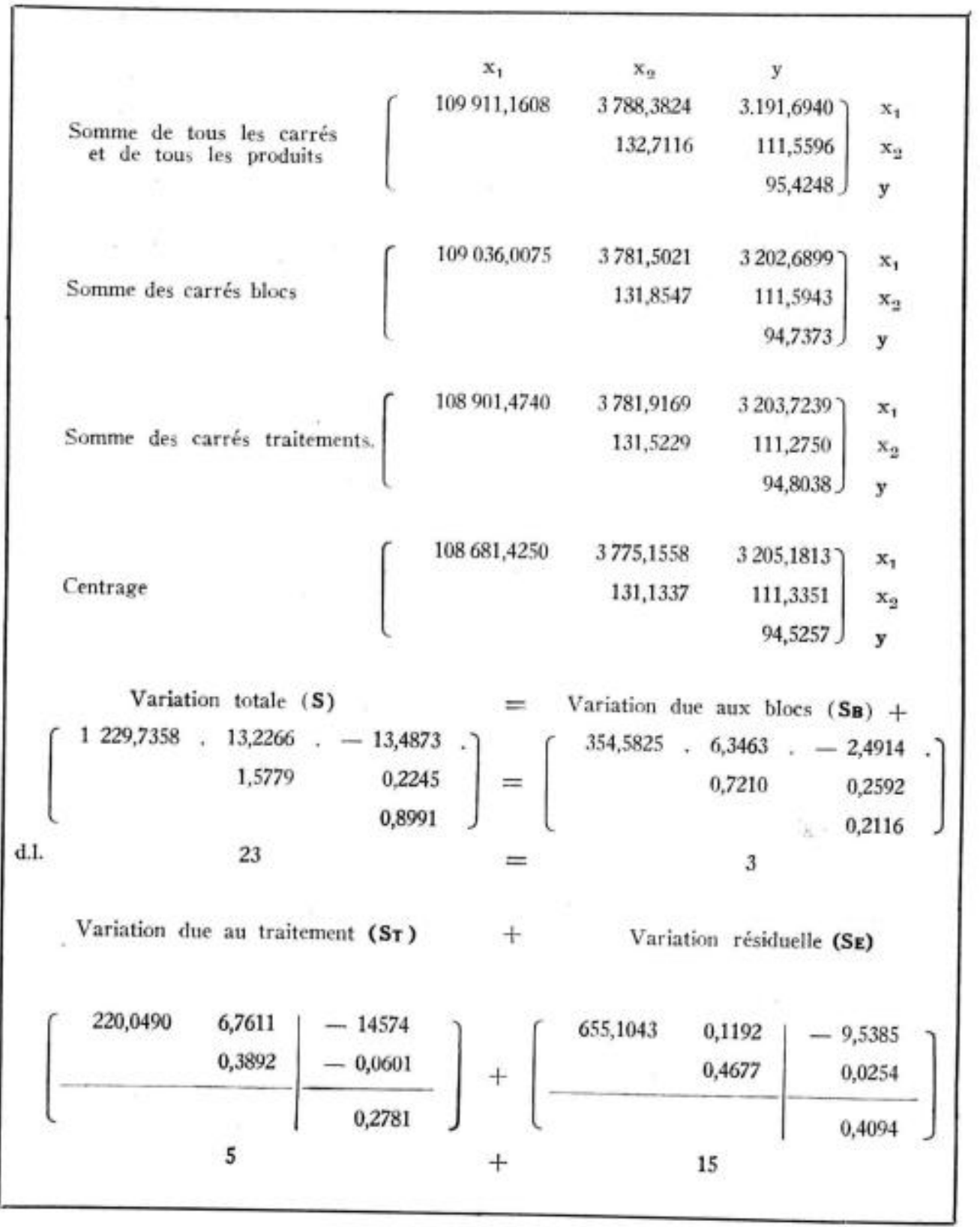

Tableau III.3.2 Analyse de covariance: $y$ affecté par deux caractères (Etude sur Lophodermisim pinastri (Schrad) Chev.) 
Toute l'analyse porte sur les moyennes des cinquante plants par parcelle unitaire qui sont indiquées au tableau III.3.1 (1).

Il est bon d'utiliser la notation matricielle dès qu'on a deux variables concomitantes à éliminer, la suite des calculs est grandement simplifiée. On a écrit au tableau III.3.2 l'égalité :

$$
\mathbf{S}=\mathbf{S}_{\mathbf{B}}+\mathbf{S}_{\mathbf{T}}+\mathbf{S}_{\mathbf{E}}
$$

la matrice $\mathbf{E}$ de la formule (II.8 bis) est incluse dans la matrice $\mathbf{S}_{\mathbf{E}}$ suivant :

$$
\mathbf{S E}=\left[\begin{array}{l|r}
\mathbf{E} & -9,5385 \\
\hline & 0,0254 \\
\hline 0,4094
\end{array}\right]
$$

Le calcul se fait sans difficulté supplémentaire.

111.3.1 Calcul de la variance résiduelle ajustée: l'équation (II.8 bis) $\mathbf{E B}-\mathbf{Q}$ se résoud par:

$$
\mathbf{B}=\mathbf{E}^{-1} \mathbf{Q}
$$

c'est-à-dire avec les données de l'expérience:

(II.8 bis) $\left[\begin{array}{rr}655,1043 & 0,1192 \\ 0,1192 & 0,4677\end{array}\right]\left[\begin{array}{l}b_{1} \\ b_{2}\end{array}\right]=\left[\begin{array}{r}-9,5385 \\ 0,0254\end{array}\right]$

soit :

$\left[\begin{array}{l}b_{1} \\ b_{2}\end{array}\right]=\left(\begin{array}{rr}0,00153 & -0,00039 \\ -0,00039 & 2,13822\end{array}\right]\left[\begin{array}{r}-9,5385 \\ 0,0254\end{array}\right]=\left(\begin{array}{r}-0,01460 \\ 0,05803\end{array}\right]$

donc la somme des carrés due à la régression est:

$$
\begin{gathered}
b_{1} P_{E}^{x y y}+b_{2} P_{E}^{x y y}=\left(\begin{array}{ll}
-0,01460 & 0,05803
\end{array}\right) \quad\left[\begin{array}{r}
-9,5385 \\
0,0254
\end{array}\right] \\
=0,1407
\end{gathered}
$$

(1) Une précision plus grande est obtenue en utilisant l'ensemble des mesures de tous les plants; mais dans ce cas on peut difficilement admettre que les variables $X_{g}$ et $\hat{Y}$ ont des distributions normales: pour la moyenne des cinquante plants le théorème de la loi forte des grands nombres permet d’affirmer que cette condition est plus surement réalisée. 
Les coefficients de régression de $\mathrm{Y}$ en $\mathrm{X}_{1}$ et de $\mathrm{Y}$ en $\mathrm{X}_{2}$ sont (1)

$$
b_{1}^{*}-\frac{-9,5385}{655,1043}=-0,01456
$$

d'oủ la part due à la régression:

$$
\begin{aligned}
& (-0,01456) \times(9,5385)=0,1389 \\
& b_{2}{ }^{*}=\frac{0,0254}{0,4677}=0,05431
\end{aligned}
$$

d'où la part due à la régression:

$$
(0,05431) \times(0,0254)=0,0014
$$

On peut alors tester l'effet séparé des deux facteurs comme il est indiqué au tableau II.5.1. On voit que l'intensité d'attaque est

\begin{tabular}{|l|c|c|c|c|}
\hline Origine de la variation & Somme des carrés & d.l. & Carré moyen & F \\
\hline Régression en $\mathrm{x}_{1}$ et $\mathrm{x}_{2}$ & 0,1407 & 2 & 0,0704 & $3,40 \mathrm{NS}$ \\
\hline $\begin{array}{l}\text { Régression en } \mathrm{x}_{1} \text { seul } \\
\text { Apport de } \mathrm{x}_{2} \text { après } \mathrm{x}_{1}\end{array}$ & $\begin{array}{c}0,1389 \\
(0,0018)\end{array}$ & $\begin{array}{l}1 \\
1\end{array}$ & 0,0018 & $0,09 \mathrm{NS}$ \\
\hline Régression en $\mathrm{x}_{2}$ seul & 0,0019 & 1 & & \\
Apport de $\mathrm{x}_{1}$ après $\mathrm{x}_{2}$ & $(0,1393)$ & 1 & 0,1393 & $6,73^{*}$ \\
\hline Frreur ajustée & $(0,2687)$ & 13 & 0,0207 & \\
\hline Erreur non ajustée & 0,4094 & 15 & & \\
\hline
\end{tabular}

Tableau III.3., Test des effets de deux caractères concomitants.

beaucoup plus influencée par la hauteur initiale du plant que par son état sanitaire avant l'expérience (2).

(1) Dans la terminologie habituelle on devait écrire:

$$
b_{y x 1, x 2}=b_{1} ; b_{x \times 2, x 1}=b_{2} ; b_{y \times 1}=b_{1}{ }^{*} ; b_{y x 2}=b_{2}{ }^{*}
$$

Pour ne pas alourdir le texte nous adoptons une notation plus simple qui, pour deux variables à éliminer, est sans ambiguité.

(2) Ce résultat est déjà très intéressant par lui-méme: s'il est confirmé on peut raisonnablement supposer que les spores qui portent l'infection se déplacent sur une faible hauteur au-dessus du niveau du sol. 
III.3.2 Test de l'effet des traitements: on effectue les mémes opérations sur la matrice $\mathbf{S}_{\mathbf{E}}+\mathbf{S}_{\mathrm{T}}$

$$
\mathrm{SE}_{\mathbf{E}}+\mathrm{ST}=\left(\begin{array}{rrr}
875,1533 & 6,8803 & -10,9959 \\
& 0,8569-0,0347 \\
& & 0,6875
\end{array}\right)
$$

On doit alors résoudre le système

$$
\mathbf{E} \mathbf{T}+\mathbf{E} \quad \mathbf{B} \mathbf{T}+\mathbf{E}=\mathbf{Q T}+\mathbf{E}
$$

done:

$$
\begin{aligned}
\mathbf{B}_{\mathbf{T}+\mathbf{E}}=\left[\begin{array}{l}
\mathrm{b}_{1}^{\circ} \\
\mathrm{b}_{2}^{\circ}
\end{array}\right] & =\left[\begin{array}{rr}
0,00122 & -0,00979 \\
-0,00979 & 1,24561
\end{array}\right]\left[\begin{array}{r}
-10,9959 \\
-0,0347
\end{array}\right] \\
& =\left(\begin{array}{r}
-0,01308 \\
0,06443
\end{array}\right]
\end{aligned}
$$

La somme des carrés due à la régression en faisant l'hypothèse que les traitements sont identiques est :

\begin{tabular}{|c|c|c|c|c|c|c|}
\hline $\begin{array}{c}\text { (1) } \\
\text { Origine } \\
\text { de la variation }\end{array}$ & $\begin{array}{c}\text { (2) } \\
\text { Somme } \\
\text { des carrés } \\
\text { non ajustec }\end{array}$ & $\begin{array}{c}\text { (3) } \\
\text { Part } \\
\text { de la } \\
\text { régression }\end{array}$ & $\begin{array}{c}(4)= \\
\text { (2) - (3) } \\
\text { Ecart } \\
\text { par rapport } \\
\text { a la } \\
\text { régression }\end{array}$ & $\begin{array}{l}\text { (5) } \\
\text { d.1. }\end{array}$ & $\begin{array}{l}(6)= \\
(4):(5) \\
\text { Carré } \\
\text { moyen }\end{array}$ & $\begin{array}{c}\text { (7) } \\
F\end{array}$ \\
\hline $\begin{array}{l}\text { Erreur E } \\
\text { Traitement+Erreur } \\
\text { E+T } \\
\text { Traitement ajusté }\end{array}$ & 0,4094 & $\begin{array}{l}0,1407 \\
0,1416\end{array}$ & $\begin{array}{l}0,2687 \\
0,5459 \\
(0,2772)\end{array}$ & $\begin{array}{r}18 \\
5\end{array}$ & $\begin{array}{l}0,0207 \\
0,0554\end{array}$ & 2,68 \\
\hline
\end{tabular}

$$
(-0,01308) \times(-10,9959)+(0,06443) \times(-0,0347)=0,1416
$$

On peut alors effectuer l'analyse de covariance proprement dite selon le modèle du tableau suivant :

Tableau III.3.4: Analyse de covariance: test de l'effet des traitements. [Etude sur Lophodermium pinastri (Schrad) Chev.]

La valeur de $\mathrm{F}$ correspond à une probabilité d'être dépassée de $10 \%$. 


\begin{tabular}{|c|c|c|c|c|c|c|c|c|}
\hline Traitements & $\bar{x}_{10}$ & $\delta x_{1}$ & $b_{1} \delta x_{1}$ & $\vec{x}_{20}$ & $8 x_{2}$ & $b_{2} \delta x_{2}$ & $\bar{y}_{10}$ & $\begin{array}{c}\hat{y}_{10}= \\
\bar{y}_{10}-b_{1} \delta x_{1}-b_{2} \delta x_{2}\end{array}$ \\
\hline 1 & 68,105 & 0,812 & $-0,012$ & 2,335 & $-0,003$ & $-0,000$ & 1,870 & 1,882 \\
\hline 2 & 71,920 & 4,632 & $-0,068$ & 2,420 & 0,082 & 0,005 & 1,880 & 1,943 \\
\hline 3 & 64,455 & $-2,838$ & 0,041 & 2,340 & 0,002 & 0,000 & 1,955 & 1,914 \\
\hline 4 & 67,110 & $-0,183$ & 0,003 & 2,460 & 0,122 & 0,007 & 1,985 & 1,975 \\
\hline$\cdot 5$ & 69,390 & 2,097 & $-0,031$ & 2,400 & 0,062 & 0,004 & 2,150 & 2,177 \\
\hline 6 & 62,775 & $-4,518$ & 0,066 & 2,070 & $-0,268$ & $-0,016$ & 2,070 & 2,020 \\
\hline Total & & 0,002 & $-0,001$ & & $-0,003$ & 0,000 & & \\
\hline Moyenne & 67,293 & & & 2,338 & & & 1,990 & 1,990 \\
\hline
\end{tabular}

Tableau III.3.5: Calcul des moyennes ajustées, [Etude sur Lophodermium pinastri (Schrad) Chev.] 
La variance d'une moyenne ajustée est par application de la formule (III.13):

$$
\begin{aligned}
\overline{\mathrm{S}}_{\hat{y}_{2}}=\frac{0,0207}{4}\left(1+\frac{1}{5}(0,00153 \times 220,0490-2 \times 0,00039 \times 6,7611+2,13822 \times 0,3892)\right. \\
=0.00638
\end{aligned}
$$

donc :

$$
s_{\xi}=0,080
$$

Le calcul des moyennes ajustées s'effectue comme aux paragraphes précédents (Tableau III.3.5.).

Au seuil de probabilité $10 \%$ on peut établir les groupements suivants (1):

$\begin{array}{ccccccc}\text { Traitements } & 1 & 3 & 2 & 4 & 6 & 5 \\ \mathfrak{z}_{10} & (1,88) & (1,91) & (1,94) & (1,98) & (2,02) & (2,18)\end{array}$

Le gain apporté par l'analyse de covariance est ici $\frac{0,0273}{0,0207}=1,32$ soit $132 \%$.

\section{4 - Un des caractères concomitants est influencé par les traitements.}

Nous avons vu ci-dessus qu'il n'était pas possible d'appliquer l'analyse de covariance rigoureusement; si on veut tout de même essayer d'appliquer les résultats énoncés plus haut, il faut être extrêmement prudent dans les conclusions (2).

Nous reprenons l'exemple étudié en III.2 de l'essai de destruction de fougère, et nous utilisons la mesure intermédiaire notée $Y$; nous n'utilisons que les matrices $\mathbf{S}_{\mathbf{E}}$ et $\mathbf{S}_{\mathbf{P}}$ et nous n'étudions que l'action des produits chimiques.

(1) Comme nous ne possédons pas de table du test de Duncan pour le seuil $10 \%$ on a simplement utilisé un test t de Student qui correspond à la plus petite différence significative de 0,20 .

(2) Cf. Cochran (W.G.), and Cox (G.M.), op. cit., p. 82-83. 


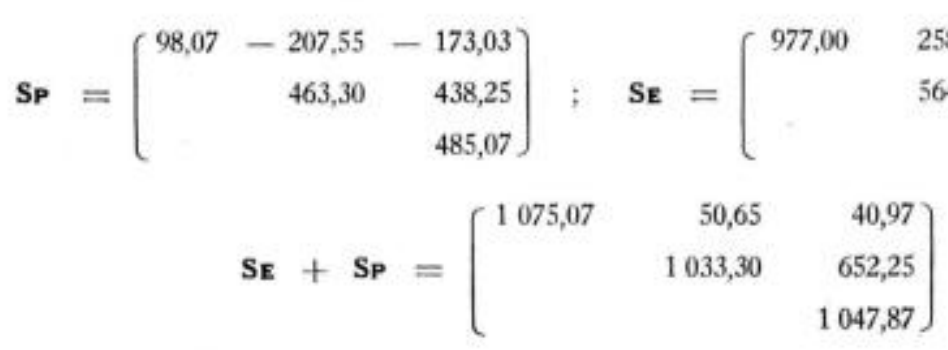

Tableau III.4.1: Matrices de dispersion nécessaires pour étudier l'effet des produits chimiques par analyse de covariance (Destruction de fougère en forêt de Granvillers).

Comme au paragraphe précédent nous calculons:

- pour la sous-matrice de $\mathbf{S}_{\mathbf{E}}$, le vecteur $\mathbf{B}=\left(\begin{array}{l}b_{1}=0,0084 \\ b_{2}=0,6309\end{array}\right)$

- pour la sous-matrice de $\mathbf{S}_{\mathbf{E}}+\mathbf{S}_{\mathbf{P}}$ le vecteur $\mathbf{B}_{\mathbf{T}+\mathbf{E}}^{\circ}=\left(\begin{array}{l}b_{1}{ }^{*}=0,0550 \\ b_{2}{ }^{*}=0,6205\end{array}\right)$

On peut alors faire l'analyse de covariance:

\begin{tabular}{|c|c|c|c|c|c|c|}
\hline $\begin{array}{c}\text { Origine } \\
\text { de la variation }\end{array}$ & $\begin{array}{c}\text { (2) } \\
\text { Somme } \\
\text { des carrés } \\
\text { non ajustée }\end{array}$ & $\begin{array}{c}\text { (3) } \\
\text { Part } \\
\text { de la } \\
\text { régression }\end{array}$ & $\begin{array}{c}(4)= \\
(2)-(3) \\
\text { Ecart } \\
\text { par rapport } \\
\text { à la } \\
\text { régression }\end{array}$ & (5) & $\begin{array}{l}(6)= \\
(4):(5) \\
\text { Carré } \\
\text { moyen }\end{array}$ & (7) \\
\hline Erreur E & 562,80 & 237,76 & 325,04 & 38 & 8,55 & \\
\hline $\begin{array}{c}\text { Traitement+Erreur } \\
E+T\end{array}$ & 1047,87 & 411,85 & 636,02 & 41 & & \\
\hline Traitement ajusté & & & 310,98 & 3 & 103,56 & $12,11^{* *}$ \\
\hline
\end{tabular}

Tableau III.4.2.: Analyse de covariance: test de l'effet des traitements (De;truction de fougère en forèt de Granvillers).

(1) Les matrices $\mathbf{E}$ et $\mathbf{E}^{-1}$ ont pour expression:

$$
\mathbf{E}=\left[\begin{array}{ll}
977,00 & 258,20 \\
258,20 & 564,00
\end{array}\right] \quad \mathbf{E}-1=\left[\begin{array}{rr}
0,0011644 & -0,0005330 \\
-0,0005330 & -0,0020171
\end{array}\right]
$$




\begin{tabular}{|c|c|c|c|c|c|c|c|c|}
\hline Produits & $x_{1 n}$ & $\delta \mathrm{x}$ & $b_{1} \delta x$ & $\bar{y}_{10}$ & $8 y$ & $\mathrm{~b}_{2} \mathrm{\delta}_{y}$ & $\bar{z}_{i 0}$ & $\begin{array}{c}z_{10}= \\
\vec{z}_{10}-b_{1} \delta x-b_{2} \delta y\end{array}$ \\
\hline AM & 16,700 & 0,975 & 0,054 & 0,100 & $-3,250$ & $-2,017$ & 1,100 & 3,063 \\
\hline RG & 13,200 & $-2,525$ & $-0,139$ & 8,400 & 5,050 & 3,134 & 10,000 & 7,725 \\
\hline RA, & 15,700 & $-0,025$ & $-0,001$ & 4,600 & 1,250 & 0,776 & 8,500 & 6,306 \\
\hline RA, & 17,300 & 1,575 & 0,087 & 0,300 & $-3,050$ & $-1,893$ & 4,500 & 7,005 \\
\hline Total & & 0,000 & 0,001 & & 0,000 & 0,000 & & \\
\hline Moyenne & 15,725 & & & & & & 6,025 & 6,025 \\
\hline
\end{tabular}

Tableau III.4.3: Calcul des moyennes ajustées (Destruction de fougère en forét de Granvillers). 
L'analyse de covariance aboutit à un test significatif; il s'agit de savoir pour pouvoir interpréter ce résultat ce qui résulte de l'élimination des deux variables concomitantes: lyse.

- l'élimination de $\mathrm{X}$ n'apporte rien de plus quà la première ana-

- en étudiant $Y$ on étudie l'effet plus ou moins durable des produits chimiques dans le temps. On peut donc après ajustement des moyennes $Z$ trouver un sens aux différences remarquées.

La variance moyenne d'une moyenne ajustée est:

$$
\begin{aligned}
S_{\gamma_{2}}= & \frac{8,55}{10}\left(1+\frac{1}{3}(98,07 \times 0,0011644+2 \times 207,55 \times 0,0005330+469,30 \times 0,0020171)\right) \\
= & 1,2204 \\
S_{i}= & 1,105
\end{aligned}
$$

Nous voyons qu'il existe un groupement simple: d'un cóté AM, de l'autre RG. RA $A_{1}$ et $\mathrm{RA}_{2}$. Nous pouvons conclure que l'effet de AM parait beaucoup plus durable que celui des autres produits. Ce résultat pouvait ètre prévu d'après la représentation schématique de la figure III.4.1: la progression entre $\mathrm{Z}$ et $\mathrm{Y}$ est moins rapide pour $\mathrm{AM}$ que pour les trois autres produits.

$$
\text { Le gain est ici de } \frac{14,07}{8,55}=1,65 \text {, done } 165 \% \text {. }
$$

\section{I11. 5 - Autres analyses classiques.}

I/I.5.1 Dispositifs orthogonaux équilibrés: c'est le cas des expériences factorielles en carré latin; l'analyse ne pose pas de difficultés nouvelles. On calcule toujours la sous-matrice $\mathbf{E}$ de $\mathbf{S}_{\mathbf{E}}$, puis le vecteur $\mathbf{B}$, c'est-à-dire les valeurs des coefficients de régression de $\mathrm{Y}$ en $\mathrm{X}_{1}, \mathrm{X}_{2}, \ldots \mathrm{X}_{\mathrm{p}}$ et enfin la somme résiduelle ajustée des carrés. Pour le test d'ensemble de l'égalité des niveaux d'un traitement on effectue les mèmes opérations sur $\mathbf{E}_{\mathbf{T}+\mathbf{E}}$

III.5.2 Cas oǹ il existe deur types derreur: ce cas est un peut plus délicat et il se présente, par exemple, dans l'interprétation d'un dispositif en "split-plot $*$. On doit alors calculer deux vecteurs régression: un pour chaque type d'erreur; d'où deux erreurs ajustées de façon différente. On peut alors effectuer l'analyse de covariance comme il est indiqué au tableau III.5.1. Le calcul des variances moyennes est alors beaucoup plus délicat (1).

(1) Cf. Ramachandran (G.), op. cit. 


\begin{tabular}{|c|c|c|c|c|c|}
\hline $\begin{array}{c}\text { Origine } \\
\text { de la variation }\end{array}$ & $\begin{array}{l}\text { Somme } \\
\text { des carrés }\end{array}$ & $\begin{array}{c}\text { Somme des carrés } \\
\text { ajustée }\end{array}$ & d.L. & $\begin{array}{c}\text { Carré moyen } \\
\text { ajusté }\end{array}$ & F \\
\hline $\begin{array}{c}\text { Blocs } \\
\text { Traitements: } \mathrm{T} \\
\text { Erreur (a) }\end{array}$ & $\begin{array}{l}Q B \\
Q \tau \\
Q r_{a}\end{array}$ & $\frac{\mathrm{D}^{2} \mathrm{~T}+\mathrm{A}}{\mathrm{D}^{2}}$ & $\begin{array}{l}1-1 \\
n_{n} \rightarrow p\end{array}$ & $\begin{array}{l}S=T \\
S=\end{array}$ & $\mathrm{S}^{2} \mathrm{~T} / \mathrm{S}^{2} \Lambda$ \\
\hline $\mathrm{T}+$ Erreur (a) & & $\mathrm{D}^{2} \mathrm{r}+\mathrm{A}$ & & & \\
\hline $\begin{array}{c}\text { Variétés: } \mathrm{V} \\
\text { Interaction } \mathrm{T} \times \mathrm{V} \\
\text { Erreur (b) }\end{array}$ & $\begin{array}{l}\text { Qv } \\
\text { QTXV } \\
Q \varepsilon_{11}\end{array}$ & $\begin{array}{l}D^{2}{ }_{v}-D^{2} \\
D T \times V ~ \\
D^{2}{ }_{b}\end{array}$ & $\mid \begin{array}{c}v-1 \\
(1-1)(v-1) \\
n_{1}-1\end{array}$ & $\begin{array}{l}S^{2} v \\
S^{2} \tau \times V \\
S^{2}\end{array}$ & 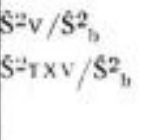 \\
\hline $\begin{array}{c}\mathrm{V}+\text { Erreur (b) } \\
\mathrm{T} \times \mathrm{V}+\text { Erreur (b) }\end{array}$ & & $\begin{array}{l}D^{2} v^{2}+1 " \\
D^{2} T x v^{2}+6\end{array}$ & & & \\
\hline Totale & $Q$ & & & & \\
\hline
\end{tabular}

Tableau III.5.1: Analyse de covariance d'un dispositif en \& split-plot s simple.

III.5.3 Dispositifs en blocs incomplets: l'interprétation se complique encore selon le modèle d'interprétation choisi (1). Si on utilise l'information entre blocs la non orthogonalité du dispositif entraine un grand nombre de difficultés; en particulier la variance d'une moyenne d'un traitement n'est pas de la forme indiquéc en (II.13) mais elle comprend deux termes nouveaux: le carré moyen Blocs-Traitements éliminés et le carré moyen intra-blocs aussi bien pour le caractère à étudier que pour ceux qu'on se propose d'éliminer (2).

\section{IV - EXTENSION DE L'ANALYSE DE COVARIANCE A DES PROBLEMES PARTICULIERS}

\section{1 - Dispositifs non orthogonaux.}

Dans des dispositifs de ce type à deux facteurs contrölés on ne peut pas interpréter l'effet d'un traitement indépendamment de l'effet d'un autre traitement. Ces cas sont tellement fréquents dans

(1) Dans un dispositif en blocs incomplets équilibrés deux modèles d'interprétation sont possibles: si on considère que la variabilité entre bloc est déterminée, les quantités représentant l'effet des blocs sont fixées; si on veut tenir compte de l'information entre blocs on doit supposer que ces mèmes quantités sont des variables aléatoires distribuées normalement. Cf. VESSEREAU (A.), op. cit., p. 359-404.

(2) Pour plus de détails sur l'interprétation, cf. Fedrker (W.T.), op. cit., p. 498-509. 
l'interprétation des dispositifs classiques qu'il faut les étudier malgré l'inconvénient que présente leur interprétation. Dans le cas d'un dispositif en blocs complets sans interaction un modèle a été proposé (1): il fait intervenir la matrice de variance-covariance des parametres des traitements. Cette dernière se calcule simplement a partir du tableau du nombre de mesures par parcelle unitaire $\left(n_{1 j}\right)$ et permet de calculer la somme des carrés traitement. A partir de ce point, on peut sans difficulté supplémentaire appliquer la méthode expliquée ci-dessus, avec les mèmes réserves que pour l'analyse des dispositifs en blocs incomplets (2).

\section{2 - Etude du rapport de deux variables.}

On veut quelquefois étudier le rapport de dęux variables dans le but d'eliminer une d'entre elles. Si au préalable on a fait une analyse de variance sur les deux facteurs, on a implicitement supposé que chacune était distribuée normalement: il est donc très dangereux de faire une analyse de variance sur le rapport car la nouvelle variable n'est absolument pas une variable normale (3). La seule solution simple consiste à faire une analyse de covariance $\mathrm{en}$ prenant comme variable indépendance la variable que l'on veut éliminer.

\section{3 - Cas où il existe un gradient de variation.}

Malgré toutes les précautions, il se peut qu'une unité de comparaison ne soit pas rigoureusement homogène, mais qu'il existe une variation indépendante des traitements, on peut essayer dans ce cas d'éliminer la variation qui s'est introduite par analyse de covariance: il suffit d'associer au caractère mesuré une variable dont les valeurs successives sont $1,2,3, \ldots$ correspondant à la position dans l'unité de comparaison (4). On peut même associer une variable plus compliquée qui ne varie pas de façon linéaire, mais comme les carrés des entiers $1,4,9, \ldots$ Dans tous les cas, il est bon d'avoir un graphique sur lequel on porte les points représentatifs en fonction de la distance pour avoir au moins une idée du gradient de variation. Pour des expériences biologiques courantes dans lesquelles les variations proviennent de différences de fertilité une variable variant linéairement suffit largement.

(1) Cf. Tochrr (K.D.), op. cit,

(2) Quand le dispositif n'est vraiment plus interprétable de façon simple, il vaut mieux, dans la majorité des cas, se ramener à une étude de régression.

(3) La loi du rapport de deux variables normales de même moyenne et de mème variance est une loi compliquéc. Pour certaines valeurs des paramètres c'est une loi de Cauchy. Cf. Agard (J.), op. cit.

(4) La variable concomitante à éliminer est donc simplement la distance à une origine fixée. 


\section{4 - Composantes de la variation.}

L'analyse de variance permet de calculer explicitement les diverses variances correspondant aux facteurs contrólés: par exemple, si on compare plusieurs provenances d'arbres composées chacune de plusieurs arbres, l'analyse de variance permet de se rendre compte sil existe des différences entre provenances. Mais on peut avoir des renseignements supplémentaires en calculant les variances entre provenances, entre arbres au sein des provenances (1). Les calculs sont simples dans les dispositif orthogonalisés, il suffit de savoir quelle est la valeur estimée par un carré moyen qui correspond à une certaine variation. Ces calculs sont aussi possibles en analyse de covariance. Par exemple dans le dispositif à un facteur contrôlé avec une seule variable concomitante:

$\mathrm{SE}^{2}=$ variance résiduelle ajustéc

$\mathrm{ST}^{2}=$ variance entre provenances $=$

$$
=\frac{1}{n}\left(\frac{Q_{T}^{y_{2}}-b_{T} Q_{T}^{z_{2}}}{t-2}-\frac{Q_{E}^{y_{2}}-b_{E} Q_{E}^{s y}}{t n-t-1}\right)
$$

oủ be est le coefficient de régression résiduelle et bT le coefficient de régression calculé à la ligne traitement dans l'analyse de covariance, $n$ est le nombre de mesures par traitement et $t$ le nombre de niveaux de traitements à comparer.

Par exemple dans l'expérience étudiée en TII.1, on a:

$$
\begin{aligned}
& \mathrm{S}_{\mathrm{E}}^{2}=5642,91 \\
& \mathrm{~S}_{\mathrm{T}}^{2}-3125,64
\end{aligned}
$$

\section{5 - Estimation de données manquantes.}

Il arrive que dans une expérience certaines données manquent, il est possible de les estimer par la méthode des moindres carrés, tous les ouvrages classiques de statistiques appliquées donnent l'expression dans le cas d'une donnée manquante (2). Il est possible de calculer ces valeurs en utilisant l'artifice de lanalyse de covariance: à chaque donnće manquante on associe une variable $\mathrm{x}$ prenant la valeur 1 si la donnée manque et 0 dans le cas contraire. Pour p données manquantes, on a donc $\mathrm{p}$ variables indépendantes prenant une des deux valeurs 0 ou 1 (3). On considère que la valeur de y cor-

(1) Ce calcul peut ètre intéressant pour des études de variation en génétique. Pour le calcul de ces variances dans d'autres plans d'expérience cf. Federer (W.T.), op. cit., p. 518-522,

(2) Cf. Vessereat (A.), op. cit., p. 239-240.

(3) On appelle la variable $\mathrm{x}$ une variable indicatrice. 
respondant à la donnée manquante est nulle. On calcule done un vecteur B, et les valeurs de y ajustées sont les valeurs

$$
\hat{y}=y_{0}-\Sigma b_{k}\left(x_{k k}-x_{o k t}\right)
$$

avec $y_{0}-0$; comme on ramène tout aux valeurs $x_{o k}=0$ et comme $\mathrm{x}_{\mathrm{k}}=1$ par définition, on a:

$$
y=-\sum_{k=1}^{k=p} b_{k}
$$

Il faut noter qu'on ne trouve pas d'autres équations que celles qu'on aurait obtenu en égalant à zéro les dérivés de la variance résiduelle $\mathrm{U}$ par rapport aux paramétres que l'on vett estimer, mais ces équations sont obtenues facilement (1).

(1) A ce sujet cf. Federer (W.T.), op. cit., p. 509-510. 


\section{BIBLIOGRAPHIE}

Agard (J.). - Mélange de deux populations normales et étude de quelques fonctions de variables normales $\mathrm{x}, \mathrm{y}$. Revue de Statistiques appliquées, 1X, 4, 1964, p. 53-70.

Cochran (W.-G.), and Cox (G.-M.). - Experimental Designs, John Wiley, New York, 1953.

Dempster (P.-A.). - Stepwise multivariate analysis of variance based on principal variables, Biometrics, $19,3,1963$, p. $478-490$.

Duguk (D.). - Traité de Statistique théorique et appliquée, Masson, Paris, 1958.

Federer (W.-T.), - Experimental Design. Theory and Application, Mac Millan, New York, 1963.

KемттноRNE (O.). - The design and analysis of experiments. John Wiley, New York, 1962.

Kendal. (M.-G.) and Sturnt (A.). - The advanced theory of statistics, Vol. 2. Griffin. Londres, 1961.

MACKEnziE (A.-M.). - The effect of thinning treatment on growth. Forestry, XXXV, 2, 1962, p. 129-138.

Monja.ion (A.). - Initiation au calcul matriciel. Vuibert, Paris, $3^{\circ}$ éd., 1961.

Ramachandrax (G.). - Analysis of covariance for the Split-plot Design average variances, Biometrics, 20, 1, 1964, p. 204-206.

RAo (C.-R.). - Advanced Statistical Methods in Biometric Research. John Wiley, New-York, 1962.

Rives (M.). - Sur la comparaison des moyennes dans les essais variétaux. Annales de 1'Amélioration des plantes, 9, 3, 1959, p. 357-376.

SNEDECOR (G.-W.). - Statistical methods. The Iowa State College Press, Ames, Iowa, 5* ed, 1956.

Tоснвк (K.-D.). - The design and analysis of block experiments. JRSS B XIV, 1952 , p. $45-100$.

Tomsssone (R.), - Méthode graphique de calculs de la moyenne et de l'écart type d'une distribution normale. Test de normalité. R.F.F, 10, 1963, p. $791-797$.

Tomassone (R.). - Application des fonctions discriminantes à des problèmes biométriques. Ann. E.N.E.F., XX, 4, 1963, p. 580-617.

Vessereau (A.). - Méthodes statistiques en biologie et en agronomie, Baillère. Paris, 1961.

Vigxal. (J.). - Caleul matriciel, Vuibert. Paris, 1961.

Wishant (J.). - Field Trials II. The Analysis of Covariance. Commonwealth Bureau of Plant Breeding and Genetics. Cambridge, 1950, 


\section{RÉSUMÉ}

Toutes les précautions prises et malgré tous les soins apportés, il n'est pas toujours possible dans la mise en place d'un dispositif expérimental de tenir compte de tous les facteurs susceptibles d'influencer le caractère étudié; la comparaison des " traitements " peut donc être faussée. L'analyse de covariance dont l'objet est de tenir compte de ces facteurs, permet d'éviter ce genre d'erreur.

Nous donnons d'abord un aperçu théorique de la méthode dans la notation matricielle, afin d'en indiquer les points délicats et les conditions exactes d'application. Nous étudions les différents tests d'hypothèses.

Ces résultats théoriques sont appliqués à des exemples d'expérimentation forestière; analyse à un facteur contrólé, analyse factorielle, dispositif en blocs complets avec deux caractères à éliminer. Nous examinons ensuite le cas délicat où un des facteurs est influencé par les traitements.

Par ailleurs, nous rattachons le problème de l'estimation des données manquantes à celui de l'analyse de covariance, ce qui permet de donner une résolution rapide à cette question. 


\section{SUMMARY}

Whatever the precautions taken and the care exercised in the setting up of experimental designs, it is not always possible to take into account all the factors likely to affect the characteristic studied; the comparison of "treatments " may therefore be distorted. Covariance analysis the object of which is to take these factors into account, helps to avoid this type of error.

First a theoretical account of the method is given in regard to matricial notation to indicate the critical points and precise conditions of its application. Various assumptive tests have been studied.

These theoretical results have been applied to forest experiments; analysis with one controled factor, factorial analysis, completeblock design with elimination of two characteristics. Then, the difficult case when one factor is affected by treatments has been examined.

Besides, the problem of missing data estimation is related to that of covariance analysis, which makes possible a rapid solution of this point. 


\section{ZUSAMMENFASSUNG}

Trotz aller ergriffenen Massnahmen und höchst zuständiger Sorgfalt, ist es nicht immer möglich in der Zusammenstellung einer Versuchsanlage, Rechnung aller Faktoren, die den ergänzten Charakter beeinflussen, zu tragen; der Vergleich der "Pflegentechnik n kann also gefälscht werden. Die Analysis der " covariance ", deren Grund die Berücksichtigung dieser Faktoren ist, gibt Gelegenheit dieses Irrtum zu vermeiden.

Wir stellen zuerst einen theoretischen Ueberblick der Matrizenbezeichnung, dar, dies im die misslichen Stellen und die genauen Anwendungsformen zu vermerken. Die verschiedene Testhypothesen werden ergründet.

Diese theoretische Ergebnisse sind beispielweise an Forstexperimenten angewendet; Analysis an einem kontrollierten Faktor, Faktorenanalysis, Zuteilung in vollständigen Teilen mit zwei auszuschneidenten Charakteren. Wir ergründen dann der misslichen Fall wobei einer der Faktoren durch die Pflegetechnik beeinflusst ist.

Anderswo, verbinden wir das Problem der Abschätzung der Fehlangaben mit dem der Covarianceanalysis, Verfahren das eine rasche Lösung dieses Falls gestattet. 